\title{
A Multilayer Micromechanical Model of the Cuticle of Curculio longinasus Chittenden, 1927 (Coleoptera: Curculionidae)
}

\author{
M. Andrew Jansen ${ }^{\mathrm{a}, *}$, Sudhanshu S. Singh ${ }^{\mathrm{b}}$, Nikhilesh Chawla ${ }^{\mathrm{c}}$, Nico M. Franz ${ }^{\mathrm{a}}$ \\ ${ }^{a}$ School of Life Sciences, Arizona State University, Tempe, Arizona, United States of America \\ ${ }^{b}$ Department of Materials Science and Engineering, Indian Institute of Technology, Kanpur, 208016, Uttar Pradesh, India \\ ${ }^{c}$ School for Engineering of Matter, Energy, and Transport, Arizona State University, Tempe, Arizona, United States of America
}

\begin{abstract}
Curculio longinasus Chittenden, 1927 (Coleoptera: Curculionidae), is a weevil species common throughout the southwestern United States that uses its rostrum - a very slender, curved, beak-like projection of the head - to excavate tunnels in plant organs (such as acorns) for egg laying (oviposition). Once the apical portion of the rostrum has been inserted into the preferred substrate for oviposition, the female begins rotating around the perimeter of the hole, elevating her head by extending the fore-legs, and rotating the head in place in a drilling motion. This action causes significant elastic deformation of the rostrum, which will bend until it becomes completely straight. To better understand the mechanical behavior of the cuticle as it undergoes deformation during the preparation of oviposition sites, we develop a comprehensive micro/macro model of the micromechanical structure and properties of the cuticle, spanning across all cuticular regions, and reliably mirroring the resultant macroscale properties of the cuticle. Our modeling approach relies on the use of multi-scale, hierarchical biomaterial representation, and employs various micromechanical schemata - e.g., Mori-Tanaka, Effective Field, and Maxwell - to calculate the homogenized properties of representative volume elements at each level in the hierarchy. We describe the configuration and behavior of this model in detail, and discuss the theoretical implications and limitations of this approach with emphasis on future biomechanical and comparative evolutionary research. Our detailed account of this approach can thereby serve as a methodological template for exploring the biomechanical behavior of new insect structures.
\end{abstract}

Keywords: Curculionidae, Beetle Cuticle, Chitin, Homogenization, Elastic Properties, Multilayer

\section{Introduction}

The purpose of this study is to establish a modeling approach to study how weevil (snout beetle) species respond to the mechanical stresses they face during the pro-

5 cess of preparing sites for oviposition (egg-laying). Our project is to create a multilayer, hierarchical, micromechanical model of insect cuticle. To do so, we mathematically characterize the microstructure of the exoskeleton of beetles in general, while using certain parameters specific 10 to an individual species, as "proof of concept" in establishing the validity of the model. The formulation of this modeling approach is a critical step towards understanding the way in which cuticular structures are optimized in form and function to accommodate mechanical loading.

${ }_{15}$ The methods employed herein will ultimately enable us to analyze the material response of an entire head to load, across multiple species. Thus, our study serves as a key element of a larger research sequence and is foundational to a future comparative, multi-taxon program.

The aims of this work are to (1) model the hierarchi- ${ }^{40}$ cal complexity of beetle cuticle to recover the bulk elastic

\footnotetext{
* Corresponding author

Email address: entojansen@gmail.com (M. Andrew Jansen)
}

constants for the different regions of cuticle, (2) verify that application of the model to a common species produces robust and valid results, and (3) didactically present the results (and more critically, the methods) to be accessible to biologists with minimal knowledge of materials science. We strongly hope that "democratizing" these methods for biologists will encourage their use more widely in studying functional morphology and will enable biologists to strengthen inferences of the function and evolution of anatomical structures. Likewise, we feel that a deeper understanding of the mechanical behavior of biological materials will ultimately permit others to unlock their potential for biomimicry. Hence, we elaborate on the use of the models and homogenization schemes herein, including their assumptions, parameters, and outputs, in an effort to make this work more accessible to other biologists.

\subsection{Biology and Rostral Adaptations of Curculio longina- sus Chittenden, 1927}

The insect species examined herein, Curculio longinasus Chittenden, 1927 (Fig. 1), is an exemplary species of the genus Curculio Linnaeus, 1758 that is common throughout the southwestern United States and northern Mexico (Gibson, 1969). Curculio is the type (name-giving) genus 

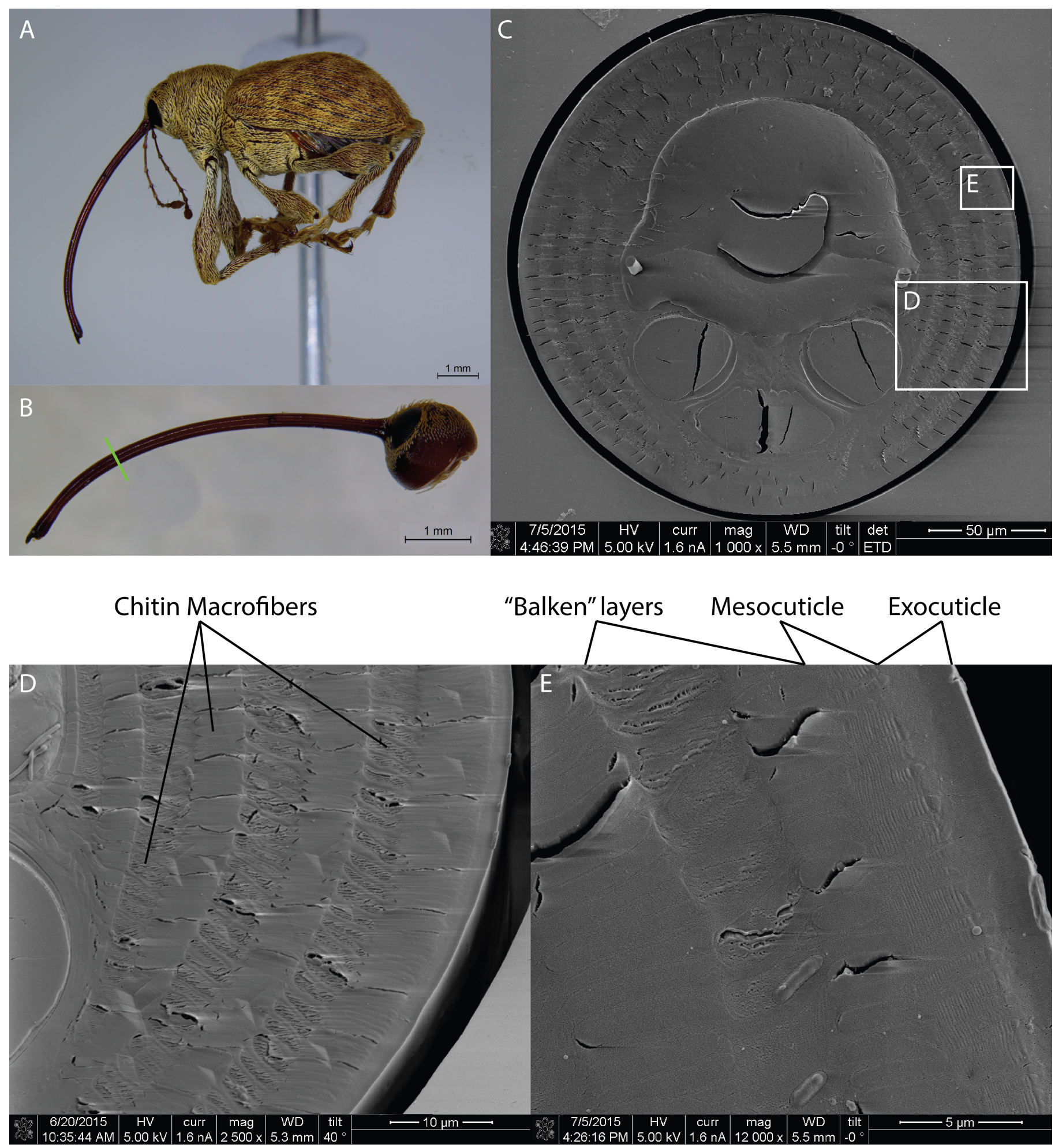

Figure 1: A Lateral habitus photograph of a female Curculio longinasus Chittenden, 1927 B Head of female C. longinasus; green line indicates location of cross-section C Scanning electron micrograph (SEM) of rostrum in cross-section; detail frames indicate size and location of subsequent magnified SEMs D Cross-section with chitin macrofibers ("balken") indicated E Cross-section with regions of cuticle indicated.

of the family Curculionidae, which are a group of beetles (order Coleoptera) commonly known as weevils. Weevils are readily identified by a structure known as the rostrum 50
(Fig. 1), which is a rigid extension of the cuticle (exoskeleton) of the head that bears the terminal chewing mouthparts (Morimoto and Kojima, 2003). Many weevils, in- 
cluding all members of genus Curculio, use the rostrum to excavate or otherwise prepare sites for oviposition (the process of laying eggs) in their respective host plants. This group is considered to be megadiverse, with over $62,000_{110}$ 55 species described to date (Oberprieler et al., 2014).

The biology of $C$. longinasus, as well as its congeners in North America, is described generally in a monographic revision by Gibson (1969), and has been expanded upon by others (Aguirre Uribe, 1978; Hughes and Vogler, 2004;

${ }_{60}$ Toju and Sota, 2006b; Toju, 2008). Many species of Curculio are impactful pest species, the most notable of which are $C$. caryae (Horn, 1873), the pecan weevil, C. elephas (Gyllenhal, 1835), the chestnut weevil, and C. nucum Linnaeus, 1758, the hazelnut weevil (Aguirre Uribe, 1978; ${ }_{65}$ Gibson, 1969). With respect to C. longinasus, Gibson (1969) lists the only confirmed host plant for this species as the southern red oak Quercus falcata Michaux, 1801. Curculio females, including those of $C$. longinasus, use their rostra to excavate a hole into the kernel of an acorn or 70 other fruiting body into which they subsequently oviposit. The developing larva feeds upon the tissue of the acorn and remains inside until pupation. During the final instar stadium, the larva will exit the acorn and burrow into the soil, where it will pupate. Eclosion generally takes place ${ }_{130}$ $75 \quad$ 2-3 weeks thereafter (Gibson, 1969).

In preparation of a site for oviposition, the adult female will excavate a bore-hole into the fruit (usually an acorn) using her rostrum and mandibles. These are unusual (and phylogenetically derived) for weevils, and in${ }_{80}$ sects in general, in that they resemble shears and articulate along a vertical plane (Gibson, 1969; Ting, 1936, 1933). The rostrum is strongly curved (up to $90^{\circ}$ ) in most species of Curculio, including C. longinasus (Fig. 1), and in some species, can exceed the length of the remainder of 85 the body (Gibson, 1969; Hughes and Vogler, 2004; Toju and Sota, 2006a,b). As the bore-hole is excavated, the female ingests the material removed by the action of the mandibles. Once the apical portion of the rostrum has been inserted into the acorn, the female begins rotating ${ }_{145}$ 90 around the perimeter of the hole and elevate her head by straightening the fore-legs. This action causes significant elastic deformation of the rostrum, which will bend until it becomes completely straight. The rostrum is kept straight by maintaining the head capsule directly above the bore- ${ }_{150}$

95 hole, and by rotating the head from side to side about the longitudinal axis of the rostrum in a (slow) drilling motion. The female will continue to move around the perimeter of the hole, using a side-stepping gait, while simultaneously maintaining an upright stance and performing the drilling

100 motion of the head, often until the entire rostrum is buried $_{155}$ in the acorn, up to the anterior portion of the eyes. This action enables the strongly curved rostrum to excavate a perfectly straight, narrow hole in the acorn (fruit), into which she may deposit one or more eggs. This behav105 ior has been described (and is easily observed) in several species of Curculio, and is likely manifested throughout ${ }^{160}$ the entire genus (Aguirre Uribe, 1978; Moffett, 1989; Toju and Sota, 2006a,b). This behavior has been recorded in the supplementary videos of Toju and Sota (2006b).

\subsection{Research Goals and Challenges}

Here we focus on the mechanical behavior of the cuticle of the $C$. longinasus rostrum as it undergoes remarkable deformation during the preparation of oviposition sites. Upon removal from the bore hole, the rostrum instantly flexes back to its original, curved shape, without any apparent damage, and in spite of the extreme degree of bending that occurs. Indeed, a single female may excavate many such holes in her adult life period, with or without eggs, and with no evident harm (Aguirre Uribe, 1978; Hughes and Vogler, 2004). The way in which this is achieved is of great interest from both a functional-mechanical and an evolutionary perspective. There has been no prior attempt to characterize the behavior of the cuticle of Curculio weevils in this context. Absent a proper material model and/or extensive mechanical testing, it is impossible to perform in-silico modelling of the rostrum during deformation to assess the response of the cuticle under load. We are therefore unable to make informed statements about the biomechanical optimization and evolution of this adaptive structure as a whole (Dumont et al., 2009).

Our principle aim is to create a micromechanical model of beetle cuticle, based on our specimens and the general structure of the cuticle, that will provide us with accurate estimates of the elastic parameters of the cuticle. Combined with a precise reconstruction of the physical dimensions of the head, this model will enable us to predict the response of the rostrum to simulated loads in future work.

Additionally, a better understanding of the physical response of this material to applied strains will permit us to eventually investigate whether the differential performance demands of regions of the weevil rostrum during oviposition are met by chemical/compositional changes in the cuticle, or by structural adjustments of the cuticular components themselves. In keeping with previous findings regarding the mechanical behavior of arthropod cuticle (see for example (Nikolov et al., 2011, 2010)), we predict that the structural arrangement and relative proportions of the components of the cuticle will most greatly affect its resulting macroscale properties. With these challenges and goals in mind, we present below a brief summary of the structure of insect cuticle and review prior research that has expanded our understanding of the mechanical behavior of this complex material.

\subsection{Microstructure of Insect Cuticle}

The insect cuticle is comprised of three general regions (Figs. 1 and 2), including (1) endocuticle, which is the most compliant and innermost region; and (3) exocuticle, which is the outermost region, usually the hardest, and has a thin waxy covering (epicuticle) that is not of great mechanical significance in most groups. Between these 


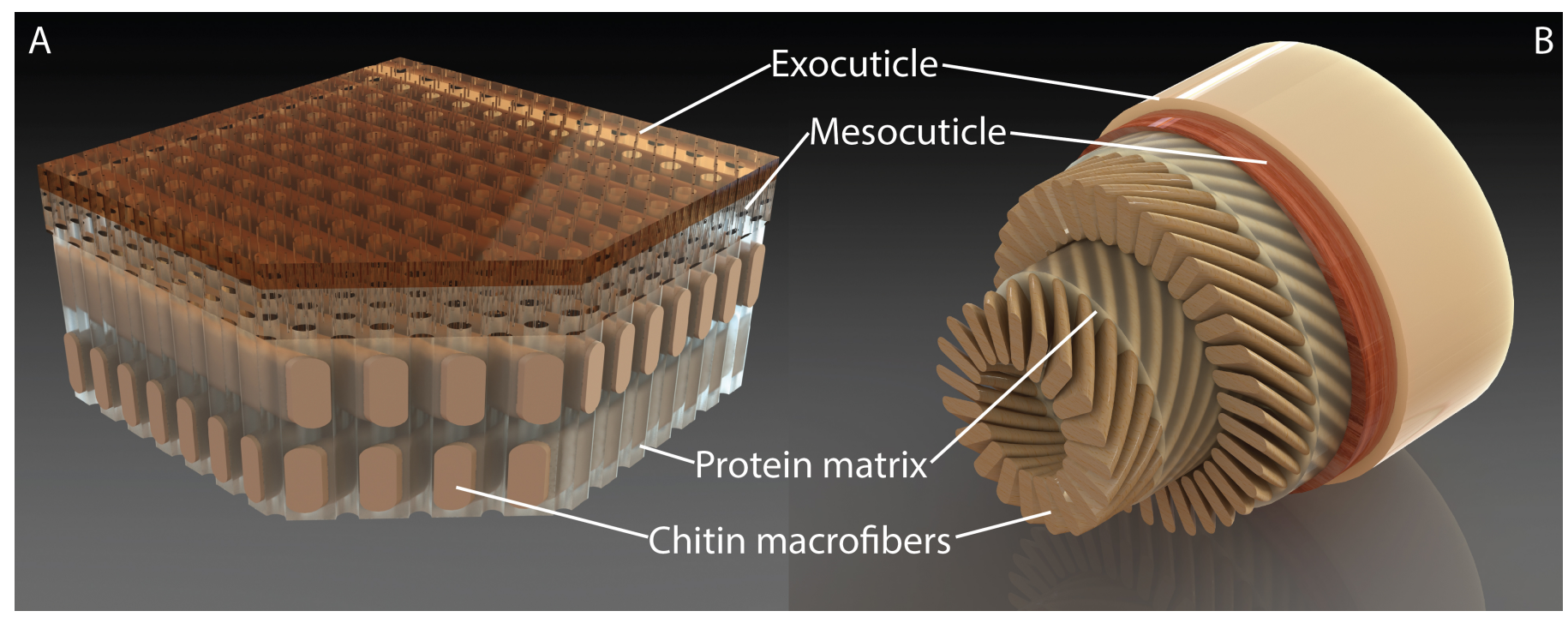

Figure 2: A Reconstruction of cuticle regions (not to scale), with pores B Manifestation of cuticle layering in cylindrical structures (as realized in the C. longinasus rostrum), without pores.

regions lies (2) the mesocuticle, which is similar to exocuticle, but is less compact and exhibits a lesser degree of tanning (sclerotization) (Klocke and Schmitz, 2011; Vincent, 1982; Vincent and Wegst, 2004). In general, these ${ }_{200}$ regions are confluent, and not necessarily sharply defined (Fig. 4). The cuticle of arthropods, including insects, is made up of chitin and numerous uncharacterized proteins, which effectively act as a matrix into which the chitin is 2004). In this way, we may consider the cuticle as a fiberreinforced composite material. Furthermore, the cuticle is laminate, and has numerous laminae of unidirectional chitin fibers embedded in a proteinaceous matrix, which are stacked at a more or less constant angle to each other ${ }_{210}$ in a helicoidal arrangement (Fig. 3). This ply arrangement is referred to as the Bouligand structure (Blackwell and Weih, 1980; Bouligand, 1972; Neville et al., 1976).

The cuticle is generally porous, with pore canals that have a twisted ribbon configuration and often a central ${ }_{215}$ pore canal fiber. These fibers likely enhance the shear stiffness of the composite by providing a form of crossstitching (Cheng et al., 2009). Beetles in particular have a highly modified endocuticle made up of large unidirectional bundles of chitin macrofibers, called "balken" (Ger- ${ }_{220}$ man for beam, strut). These layers are generally pseudoorthogonal (Figs. 2 and 5), although some other configurations have been observed (Hepburn and Ball, 1973; van de Kamp and Greven, 2010). A thorough review of the balken structure can be found in the work of van de Kamp and ${ }_{225}$ Greven (2010) and van de Kamp et al. (2015). In addition to its micro-structural properties, the cuticle is also strongly influenced by its chemical properties, especially the degree of tanning and overall water content, as discussed in great detail by Klocke and Schmitz (2011) and $_{230}$ Vincent and Wegst (2004).

\subsection{Review of Prior Studies and Modeling Approach}

Prior mechanical simulation studies of the arthropod exoskeleton and the insect cuticle as highly structured, hierarchically organized biomaterials have greatly advanced our knowledge of the behavior of these structures. Of particular significance is the modeling approach of (Nikolov et al., 2011, 2010) as applied to the endocuticle of Homarus americanus Edwards, 1873. We emulate this approach for modeling hierarchical materials using the micro/macro method (see below). Some prior analyses of the arthropod cuticle have failed to consider the layering of the exoskeleton, and have furthermore posited (for pragmatic modeling reasons) that the cuticle is homogeneously isotropic, thereby neglecting the mechanical implications of its microstructure (Flannigan, 1998; Herbert et al., 2000; Hörnschemeyer et al., 2013; Mccullough et al., 2014). However, in actuality the cuticle is a highly complex, hierarchically layered, and textured material, whose configuration and properties can vary within an individual organism and across species or higher-level taxa, and which displays a correspondingly complex response to mechanical loads (Raabe et al., 2005; Vincent, 1982). Our ability to apply previous material models to simulate this behavior is limited by our knowledge of the exact makeup of the cuticle, and especially knowledge of the relative water and protein content, which generally prohibits modeling of the viscoelastic response of this material (Nikolov et al., 2011, 2010). All mechanical simulations of the cuticle (including this work) have thus far been limited to conditions of small strain and assume linear elasticity.

We adopt the micro/macro method to achieve balance between model complexity and performance. To explain our modeling approach, we first outline the basic principles of the micro/macro method. We then develop the specific formulation, notation, and relevant assumptions 


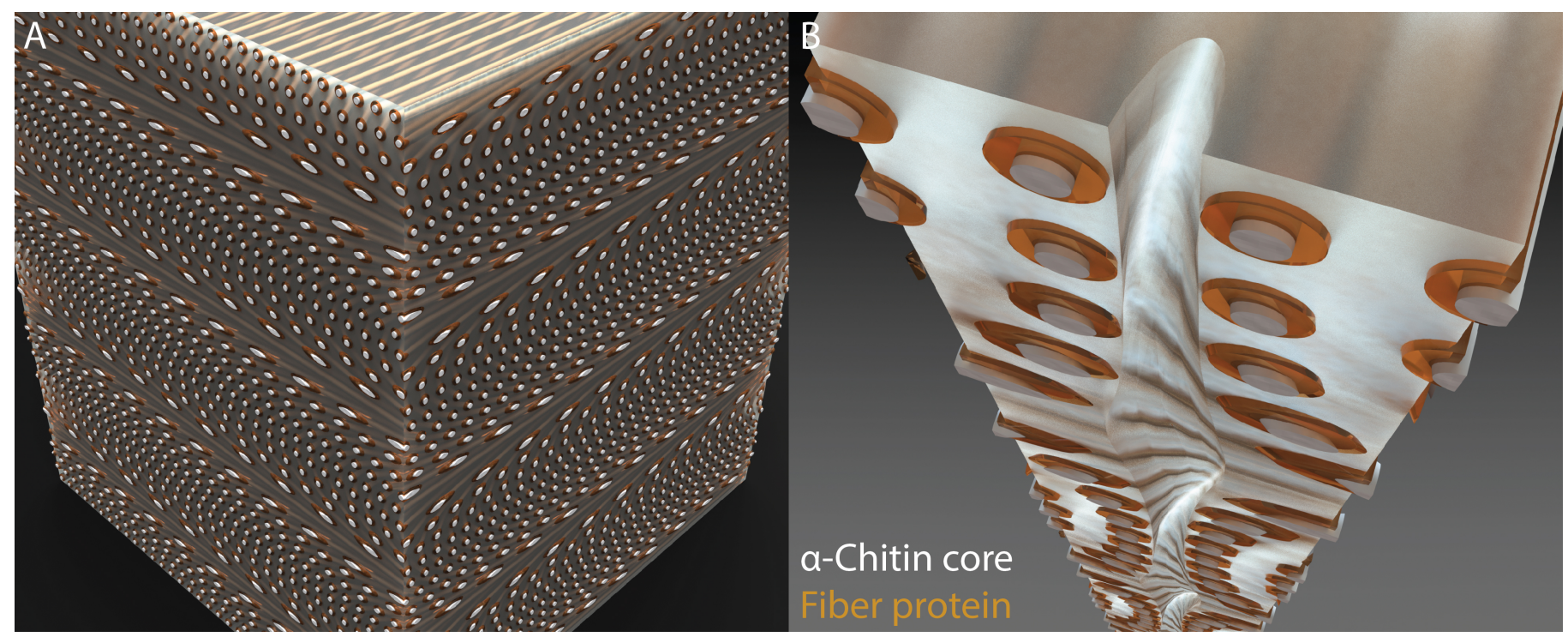

Figure 3: A Reconstruction of Bouligand structure of mesocuticle (approx. to scale), without pore canals B Typical "twisted-ribbon" pore canal in mesocuticle (bisected), without pore canal fiber; in reality, pores are often larger with nanofibrils wrapping around canals.

of the models used to calculate the effective properties of the different regions of the cuticle observed in our speci-265 mens. Considerable space is allocated to explain our modeling approach for each region of the rostrum, focusing on decisions related to the inclusion and specific configuration of parameters due either to mathematical contingencies, input from from relevant literature, or experimental observations. We thoroughly characterize the outputs ${ }_{270}$ al level of complexity, with the aim to reach both biologist and biomechanical engineering audiences, both of which might find our methods relevant to their respective research programs.

\section{Methods}

\subsection{Micro/Macro Method}

The micro/macro approach enables us to take into account the integrated rostrum microstructure to calculate its overall macroscopic properties (Doghri, 2000; Mura, Nikolov et al., 2011, 2010). This the use of representative volume elements (RVE), which280 can be considered as the smallest volume of material that has the same mechanical properties as the material at the macroscale (Doghri, 2000). In composite materials, a single RVE shows a heterogeneous composition, having two or more constituents with different properties (Reddy, 2004). The effective macroscale properties of the RVE are calculated using an appropriate homogenization scheme that ${ }^{285}$ takes into account the relevant material properties of each component, and yields the mechanical properties of the RVE as if it were of homogeneous composition (Doghri, 2000; Mura, 1987). By describing the homogenized properties of a RVE for each level of microstructure in the cu- ticle, we are able to find the bulk mechanical properties of the different types of cuticle present in the exoskeleton.

\subsection{Symbols and Notation}

In this section we summarize the definitions of terms and symbols used throughout this study. Additional information for parameters specific to each model are provided in subsequent sections.

$E_{i}$ Young's modulus of elasticity along axis $i$

$\nu_{i j}$ Poisson's ratio corresponding to displacement along axis $j$ under load along axis $i$

$G_{i j}$ Shear modulus along axis $j$ in the plane normal to axis $i$

$K$ Bulk modulus

$\theta$ Angle of rotation

$V$ Volume

$n$ Number of constituent elements within representative volume element (RVE)

$\varphi_{e}$ Volume fraction of constituent element $e$ (e.g. matrix or fiber)

$r_{f}$ Fiber radius

$s_{f}$ Lateral separation distance of fibers embedded in a matrix

$C_{m}$ Fourth-order stiffness tensor of a material $m$

$S_{m}$ Used to represent either the fourth-order compliance tensor or the Eshelby's tensor of a material $m$, as specified in the respective context. 
$I$ Identity matrix

$R$ Rotation matrix

$T_{i j k l}^{\alpha}$ Elements of standard tensor basis

$H_{i}$ Fourth-order compliance contribution tensor for an inhomogeneity $i$

$P_{m}$ Fourth-order Hill's tensor of a material $m$ (typically used for fibers embedded in a matrix)

$Q_{m}$ Fourth-order Hill's tensor of a material $m$ (typically ${ }_{345}$ used for voids embedded in a matrix)

300 : Indicates the tensor product contracted over two indices

$M^{-1}$ Indicates the inverse of a matrix $M$

$M^{T}$ Indicates the transpose of a matrix $M$

\subsection{Homogenization Schemes and Limitations}

In this section, we specify the models and homogenization schemes utilized to calculate the effective properties of the weevil rostrum cuticle. We list each in order of its simplicity and appearance in the study. For each, we $\mathrm{e}^{350}$ give a summary of the parameters of the model, conditions under which the model may be considered valid, and the six models presented herein can be classified as belonging to one of three higher "families" of models: (1) numer-355 ical/volumetric averaging of RVEs, specifically the Voigt model and isotropic approximation of anisotropic media; (2) homogenization of a single inclusion (or multiple noninteracting inclusions) in a matrix, specifically the MoriTanaka model and non-interaction approximation; and (3) 360 homogenization of multiple interacting inclusions in a matrix, specifically the effective field method ${ }^{1}$ and Maxwell scheme. The application of each method is discussed in Section 3.

\subsubsection{Voigt Model}

Assumptions: The Voigt model assumes that an imposed macro strain will result in a uniform strain field within

${ }^{1}$ Note that this collapses to a specific subset of possible solutions ${ }^{380}$ that coincides with the Mori-Tanaka model, when the "correlation hole" is concentric and of the same shape as the inclusions. resulting from strain imposed on a material) so that the macro strain is equal to the average strain within the RVE (Doghri, 2000). We calculate the effective tensor, $C_{e f f}$, of an RVE by taking the volumetric average of the stiffness tensors of the individual constituents of the RVE.

Parameters: The following are parameters specific to this model.

$C_{\text {eff }}$ Effective stiffness tensor of the RVE

$V_{i}$ Volume of the $i^{\text {th }}$ element of the RVE

$V_{R V E}$ Volume of the RVE

$C_{i}$ Stiffness tensor of the $i^{\text {th }}$ element of the RVE

Formulation: Below we present the formulation of this model:

$$
C_{\text {eff }}=\sum_{i=1}^{n} \frac{V_{i}}{V_{R V E}} C_{i}
$$

\subsubsection{Mori-Tanaka Model}

Assumptions: The Mori-Tanaka model was developed for to calculate the effective properties of two phase composites containing dilute, aligned, ellipsoidal inclusions, under conditions of linear elasticity, where the inclusions have the same properties as the matrix and are isotropic (Mori and Tanaka, 1973; Mura, 1987). This model generally treats each inclusion as if it were isolated in an infinite matrix so that the inclusions do not interact with each other, and thus produces optimal results when the volume fraction of inclusions is less than 20-30\% (Doghri, 2000; Nikolov et al., 2011). See also Buryachenko et al. (2008) for problems associated with this method. When the inclusions have different properties than the matrix material, they are referred to as inhomogeneities, and may be simulated by an equivalent inclusion with the properties of the matrix with a uniform eigenstrain, using the equivalent inclusion method (Benveniste, 1987; Mura, 1987).

By incorporating the equivalent inclusion method, a reformulation of the Mori-Tanaka model was created to accommodate the calculation of the effective properties of transversely orthotropic or anisotropic inclusions (such as cracks or fibers) embedded in an isotropic matrix, depending solely on the properties of the matrix material and the shape of the inhomogeneities (Benveniste, 1987). We therefore assume that the material is a linear elastic, two-phase composite with perfect bonding between an isotropic matrix material and the inclusions, which are identical, dilute, aligned, and ellipsoidal in shape.

Parameters: The following are parameters specific to this model. 
$C_{\text {eff }}$ Effective stiffness tensor of the RVE

$\varphi_{M}$ Volume fraction of the isotropic matrix

$\varphi_{F}$ Volume fraction of the fibrous inhomogeneities ${ }_{405}$

$\nu_{M}$ Poisson's ratio of the isotropic matrix

$C_{M}$ Stiffness tensor of the isotropic matrix

$C_{F}$ Stiffness tensor of the fibrous inhomogeneities

$A_{F}$ Strain concentration factor for the fibrous inho-410 mogeneities

$S_{F}$ Eshelby's tensor for a cylindrical inclusion

Formulation: Below we present the formulation of this model.

$C_{e f f}=C_{M}+\varphi_{F}\left[\left(C_{F}-C_{M}\right): A_{F}\right]\left[\varphi_{M} I+\varphi_{F} A_{F}\right]^{-1}$

Where:

$$
A_{F}=\left[I+S_{F}:\left(C_{M}\right)^{-1}:\left(C_{F}-C_{M}\right)\right]^{-1}
$$

For an infinite isotropic elastic matrix, with a single embedded circular cylinder, whose longitudinal axis is aligned with axis 1, the non-zero components of the Eshelby's tensor, $S_{F}$, are as follows:

$$
\begin{gathered}
S_{2211}=S_{3311}=\frac{\nu_{M}}{2\left(1-\nu_{M}\right)} \\
S_{2222}=S_{3333}=\frac{1}{2\left(1-\nu_{M}\right)}\left[\frac{3}{4}+\frac{\left(1-2 \nu_{M}\right)}{2}\right] \\
S_{2233}=S_{3322}=\frac{1}{2\left(1-\nu_{M}\right)}\left[\frac{1}{4}-\frac{\left(1-2 \nu_{M}\right)}{2}\right] \\
S_{2323}=\frac{1}{2\left(1-\nu_{M}\right)}\left[\frac{1}{4}+\frac{\left(1-2 \nu_{M}\right)}{2}\right] \\
S_{1212}=S_{1313}=\frac{1}{4} \\
\text { *note that: } S_{1111}=S_{1122}=S_{1133}=0
\end{gathered}
$$

Where:

$$
S_{F}=\left[\begin{array}{cccccc}
S_{1111} & S_{1122} & S_{1133} & 0 & 0 & 0 \\
S_{2211} & S_{2222} & S_{2233} & 0 & 0 & 0 \\
S_{3311} & S_{3322} & S_{3333} & 0 & 0 & 0 \\
0 & 0 & 0 & 2 S_{2323} & 0 & 0 \\
0 & 0 & 0 & 0 & 2 S_{1313} & 0 \\
0 & 0 & 0 & 0 & 0 & 2 S_{1212}
\end{array}\right]
$$

\subsubsection{Non-Interaction Approximation}

Assumptions: For our purposes, the non-interaction approximation is similar to the Mori-Tanaka model in its assumptions; namely, that we are finding the efposite containing a matrix phase and identical inclusions that are ellipsoidal in shape and do not interact with each other (Kachanov, 1999; Sevostianov and Kachanov, 2002; Sevostianov et al., 2001, 2005). As in the Mori-Tanaka model, this method simulates the effect of adding a single inclusion to the matrix

material, thus assuming no interaction between inclusions. The effective properties of the composite are assumed to be equal to the sum of the stiffness (or compliance) of the matrix and the effect of the inclusions either as a stiffness or compliance contribution tensor, respectively (Kachanov, 1999; Sevostianov et al., 2005). Because we use this method in a matrix that is transversely isotropic, with inclusions (specifically pores) whose longitudinal axes are aligned with the axis of transverse isotropy, we use the formulation of Sevostianov et al. (2005), which utilizes explicit inversions made simpler by representation of fourth-order tensors in "tensorial basis", as indicated below. See Kachanov (1999) and Sevostianov et al. (2005) for more information.

Parameters: The following are parameters specific to this model.

$S_{\text {eff }}$ Effective compliance tensor of the RVE

$S_{M}$ Compliance tensor of the matrix

$V_{i}$ Volume of the $i^{\text {th }}$ inhomogeneity

$V_{R V E}$ Volume of the RVE

$\varphi_{I}$ Volume fraction of the inclusions

$H_{i}$ Compliance contribution tensor of the $i^{\text {th }}$ inhomogeneity

$H_{N I}$ Compliance contribution tensor of all inhomogeneities for the non-interaction model

$h_{\alpha}$ Components of compliance contribution tensor $H_{i}$ in standard tensor basis

$C_{M}$ Stiffness tensor of the matrix

$C_{\alpha}$ Components of $C_{M}$ expressed in standard tensor basis

$T_{i j k l}^{\alpha}$ Tensors for expression in standard tensor basis

Formulation: Below we present the formulation of this model.

In general, the non-interaction approximation for a two-phase composite is given by:

$$
S_{e f f}=S_{M}+H_{N I}
$$

Where $H_{N I}$ is given by the sum of the compliance contribution tensors for $n$ inhomogeneities in the RVE, such that:

$$
H_{N I}=\sum_{i=1}^{n} H_{i}
$$

The $i^{\text {th }}$ inhomogeneity has a compliance contribution tensor $H_{i}$, expressed in tensorial basis as:

$$
H_{i}=\frac{V_{i}}{V_{R V E}} \sum_{\alpha=1}^{6} h_{\alpha} T_{i j k l}^{\alpha}
$$


By substitution of Eq. (8) into Eq. (7):

$$
H_{N I}=\sum_{i=1}^{n}\left(\frac{V_{i}}{V_{R V E}} \sum_{\alpha=1}^{6} h_{\alpha} T_{i j k l}^{\alpha}\right)
$$

However, assuming all inhomogeneities are identical, this can be expressed as:

$$
H_{N I}=\frac{n V_{i}}{V_{R V E}} \sum_{\alpha=1}^{6} h_{\alpha} T_{i j k l}^{\alpha}
$$

Therefore, because $\frac{n V_{i}}{V_{R V E}}=\varphi_{I}$ :

$$
H_{N I}=\varphi_{I} \sum_{\alpha=1}^{6} h_{\alpha} T_{i j k l}^{\alpha}
$$

If we assume the general form of a $6 \times 6$ matrix, then a symmetrical tensor $M$, expressed as a $6 \times 6$ matrix, has the following components (Doghri, 2000):

$$
M=\left[\begin{array}{llllll}
M_{1111} & M_{1122} & M_{1133} & M_{1123} & M_{1113} & M_{1112} \\
M_{2211} & M_{2222} & M_{2233} & M_{2223} & M_{2213} & M_{2212} \\
M_{3311} & M_{3322} & M_{3333} & M_{3323} & M_{3313} & M_{3312} \\
M_{2311} & M_{2322} & M_{2333} & M_{2323} & M_{2313} & M_{2312} \\
M_{1311} & M_{1322} & M_{1333} & M_{1323} & M_{1313} & M_{1312} \\
M_{1211} & M_{1222} & M_{1233} & M_{1223} & M_{1213} & M_{1212}
\end{array}\right]
$$

Any symmetrical fourth-order tensor expressed with components expressed in $6 \times 6$ matrix form can also be represented in a "standard tensor basis" as:

$$
M=\sum_{\alpha=1}^{6} M_{\alpha} T_{i j k l}^{\alpha}
$$

Where $M_{\alpha}$ are of the same form as $C_{\alpha}$, given in Eqs. (16), and the non-zero components of tensors 445 $T_{i j k l}^{\alpha}$ are as follows:

$$
\begin{gathered}
T_{1111}^{1}=T_{2222}^{1}=T_{1122}^{1}=T_{2211}^{1}=1 \\
T_{1212}^{2}=T_{2121}^{2}=T_{1221}^{2} \\
=T_{2112}^{2}=T_{1111}^{2}=T_{2222}^{2}=\frac{1}{2} \\
T_{1122}^{2}=T_{2211}^{2}=-\frac{1}{2} \\
T_{1133}^{3}=T_{2233}^{3}=1 \\
T_{3311}^{4}=T_{3322}^{4}=1 \\
T_{1313}^{5}=T_{2323}^{5}=T_{1331}^{5}=T_{2332}^{5}=T_{3113}^{5} \\
=T_{3223}^{5}=T_{3131}^{5}=T_{3232}^{5}=\frac{1}{4} \\
T_{3333}^{6}=1
\end{gathered}
$$

The coefficients $h_{\alpha}$ are:

$$
\begin{gathered}
h_{1}=\frac{2 C_{3} C_{4}-C_{6}\left(2 C_{1}+C_{2}\right)}{4 C_{2}\left(C_{3} C_{4}-C_{1} C_{6}\right)} \\
h_{2}=\left(\frac{1}{C_{1}}+\frac{2}{C_{2}}\right) \\
h_{3}=h_{4}=\frac{C_{3}}{2\left(C_{3} C_{4}-C_{1} C_{6}\right)} \\
h_{5}=\frac{8}{C_{5}} \\
h_{6}=\frac{C_{1}}{C_{3} C_{4}-C_{1} C_{6}}
\end{gathered}
$$

Where $C_{\alpha}$ are given in terms of the $6 \times 6$ matrix components of the stiffness tensor $C_{M}$ such that:

$$
\begin{gathered}
C_{1}=\frac{C_{1111}+C_{1122}}{2} \\
C_{2}=2 C_{1212} \\
C_{3}=C_{1133} \\
C_{4}=C_{3311} \\
C_{5}=4 C_{1313} \\
C_{6}=C_{3333}
\end{gathered}
$$

\subsubsection{Effective Field Method}

Assumptions: With the effective field method, all preceding assumptions for the non-interaction approximation are applicable, except the inhomogeneities are expected to interact with each other (the interaction is assumed to be geometrical, see Sevostianov (2014)). This method simulates the interaction between inclusions by placing them into an local strain field. Additionally, it is assumed, for simplicity, that the strain field of each inclusion is identical to that of any other inclusion, and that this field does not depend on the geometry or elastic properties of the inclusions, but rather, is determined by the elastic properties of the matrix and orientation of the inclusions (Sevostianov et al., 2005). By additionally assuming that there is symmetry to the distribution of inhomogeneities, and that the "correlation hole" (the region within which it is unlikely that the centers of other inclusions will be found) is of the same shape and concentric with the inhomogeneity, it is possible to provide an explicit solution for this method that coincides with the Mori-Tanaka Model (Sevostianov et al., 2005). This is the case if the inclusions under consideration are aligned, identical, and arranged such that the distance between any two neighboring fibers is identical (Buryachenko et al., 2008). We use the formulation of Sevostianov et al. (2005), which utilizes explicit inversions made simpler by representation of fourth-order tensors in "tensorial basis", in the form indicated by Eq. (13). It is assumed that axis 3 is the axis of transverse 
isotropy, aligned with the axes of rotation of the inclusions.

Parameters: The following are parameters specific to this model.

$C_{\text {eff }}$ Effective stiffness tensor of the RVE

$C_{M}$ Stiffness tensor of the matrix

$C_{\alpha}$ Components of $C_{M}$ expressed in standard tensor basis

$C_{D}$ Inverse of $S_{D}$

$C_{D}^{\alpha}$ Components of $C_{D}$ expressed in standard tensor basis

$S_{I}$ Compliance tensor of an inclusion

$S_{M}$ Compliance tensor of the matrix

$S_{D}$ Difference of the compliance tensors of the inclusions and matrix, respectively

$\varphi_{I}$ Volume fraction of the inclusions

$\varphi_{M}$ Volume fraction of the matrix

$P$ Hill's tensor

$p_{\alpha}$ Components of $P$ expressed in standard tensor basis

$\Delta_{1}$ Coefficient of tensor inversion defined in Eq. (19)

$\Delta^{*}$ Coefficient of tensor inversion defined in Eq. $(20)_{495}$ $T_{i j k l}^{\alpha}$ Tensors for expression in standard tensor basis

Formulation: Below we present the formulation of this model.

$$
C_{e f f}=\sum_{\alpha=1}^{6} C_{e f f}^{\alpha} T_{i j k l}^{\alpha}
$$

Where $C_{e f f}^{\alpha}$ are the components of $C_{e f f}$ given by:

$$
\begin{gathered}
C_{e f f}^{1}=C_{1}+\frac{\varphi_{I}}{2 \Delta^{*}}\left[\frac{2 C_{D}^{1}}{\Delta_{1}}+\varphi_{M} P_{6}\right] \\
C_{e f f}^{2}=C_{2}+\varphi_{I}\left[\frac{1}{C_{D}^{2}}+\varphi_{M} P_{2}\right]^{-1} \\
C_{e f f}^{3}=C_{e f f}^{4}=C_{3}+\frac{\varphi_{I}}{\Delta^{*}}\left[\frac{C_{D}^{3}}{\Delta_{1}}-\varphi_{M} P_{3}\right] \\
C_{e f f}^{5}=C_{5}+4 \varphi_{I}\left[\frac{4}{C_{D}^{5}}+\varphi_{M} P_{5}\right]^{-1} \\
C_{e f f}^{6}=C_{6}+\frac{\varphi_{I}}{2 \Delta^{*}}\left[\frac{2 C_{D}^{6}}{\Delta_{1}}+\varphi_{M} P_{1}\right]
\end{gathered}
$$

Where $^{2}$ :

$$
\Delta_{1}=2\left[C_{D}^{1} C_{D}^{6}-C_{D}^{3} C_{D}^{4}\right]
$$

\footnotetext{
${ }^{2}$ Note that in Sevostianov et al. (2005), this is erroneously produced as: $\Delta_{1}=C_{D}^{1} C_{D}^{6}-\left(C_{D}^{3}\right)^{2}$; see Sevostianov et al. (2015), Eq. A.4, for the correct formulation of $\Delta$ for tensor inversion in tensorial basis.
}

$$
\begin{aligned}
\Delta^{*}=2\left[\frac{C_{D}^{6}}{2 \Delta_{1}}+\varphi_{M} P_{1}\right] & {\left[\frac{2 C_{D}^{1}}{\Delta_{1}}+\varphi_{M} P_{6}\right]-} \\
& 2\left[\frac{C_{D}^{3}}{\Delta_{1}}+\varphi_{M} P_{3}\right]^{2}
\end{aligned}
$$

Given:

$$
C_{D}=C_{I}-C_{M}
$$

Additionally, $C_{D}^{\alpha}$ and $C_{\alpha}$ are components of the stiffness tensors $C_{D}$ and $C_{M}$, respectively, that may be derived from Eqs. (16). Furthermore, $p_{\alpha}$ are the components of Hill's tensor, and are given by:

$$
\begin{gathered}
p_{1}=\frac{1}{2\left(2 C_{1}+C_{2}\right)} \\
p_{2}=\frac{1}{2\left(2 C_{1}+C_{2}\right)}+\frac{1}{C_{2}} \\
p_{3}=p_{4}=p_{6}=0 \\
p_{5}=\frac{2}{C_{5}}
\end{gathered}
$$

\section{Assumptions: For slightly anisotropic media, it is possi-} ble to create an isotropic approximation of the material from its elastic constants (Saadat et al., 2012; Sevostianov and Kachanov, 2008). For this particular formulation, we assume that the material in question is transversely isotropic.

Formulation: Below we present the formulation of this model. From Hook's Law, we may derive the elastic constants of orthotropic media from their stiffness and compliance tensors as follows:

$$
S_{m}=\left[C_{m}\right]^{-1}=
$$

$$
\left[\begin{array}{cccccc}
\frac{1}{E_{1}} & -\frac{\nu_{21}}{E_{2}} & -\frac{\nu_{31}}{E_{3}} & 0 & 0 & 0 \\
-\frac{\nu_{12}}{E_{1}} & \frac{1}{E_{2}} & -\frac{\nu_{32}}{E_{3}} & 0 & 0 & 0 \\
-\frac{\nu_{13}}{E_{1}} & -\frac{\nu_{23}}{E_{2}} & \frac{1}{E_{3}} & 0 & 0 & 0 \\
0 & 0 & 0 & \frac{1}{G_{23}} & 0 & 0 \\
0 & 0 & 0 & 0 & \frac{1}{G_{31}} & 0 \\
0 & 0 & 0 & 0 & 0 & \frac{1}{G_{12}}
\end{array}\right]
$$

For a transversely isotropic material, the isotropic approximations of the shear and bulk moduli may be calculated, respectively, as:

$$
\begin{gathered}
G^{-1}=4\left[\left(1+\nu_{12}\right) E_{1}^{-1}+\left(1+\nu_{23}\right) E_{2}^{-1}+\left(1+\nu_{31}\right) E_{3}^{-1}\right] \\
+3\left(G_{12}^{-1}+G_{23}^{-1}+G_{31}^{-1}\right) \\
K^{-1}=\left(1-2 \nu_{12}\right) E_{1}^{-1}+\left(1-2 \nu_{23}\right) E_{2}^{-1}+\left(1-2 \nu_{31}\right) E_{3}^{-1}
\end{gathered}
$$

From these, we may also find the isotropic approximations of Poisson's ratio and Young's modulus:

$$
\begin{gathered}
\nu=\frac{3 K-2 G}{2(3 K+G)} \\
E=\frac{9 K G}{3 K+G}
\end{gathered}
$$


The stiffness tensor of the isotropic approximation may then be calculated, by Hook's Law, such that:

$$
\begin{gathered}
C=\frac{E}{(1+\nu)(1-2 \nu)} \times \\
{\left[\begin{array}{cccccc}
(1-\nu) & \nu & \nu & 0 & 0 & 0 \\
\nu & (1-\nu) & \nu & 0 & 0 & 0 \\
\nu & \nu & (1-\nu) & 0 & 0 & 0 \\
0 & 0 & 0 & \frac{(1-2 \nu)}{2} & 0 & 0 \\
0 & 0 & 0 & 0 & \frac{(1-2 \nu)}{2} & 0 \\
0 & 0 & 0 & 0 & 0 & \frac{(1-2 \nu)}{2}
\end{array}\right]}
\end{gathered}
$$

\subsubsection{Maxwell Homogenization Scheme}

Assumptions: For the Maxwell homogenization scheme, we observe the same assumptions shared between the previous models. The approach of Maxwell was to remove a domain $\Omega$ from a composite material and insert it into the matrix, where its effect will invariably be described by either the sum of the compliance (or stiffness) contribution tensors of the inclusions, or by the compliance (or stiffness) contribution tensor of the entire domain, which is assumed to have unknown properties. By equating these quantities, it is possible, given the aforementioned assumptions of our system, to explicitly calculate the effective properties of $\Omega$ as our RVE, while accounting for the interactions between inhomogeneities (Sevostianov and Giraud, 2013; Sevostianov, 2014; Sevostianov et al., 2015; Vilchevskaya and Sevostianov, 2015). We follow the formulation of Sevostianov (2014), which has been successfully used to calculate the effective properties of cortical bone, another biomaterial with a complex microstructure (Vilchevskaya and Sevostianov, 2015).

Parameters: The following are parameters specific to this model.

$S_{\text {eff }}$ Effective compliance tensor of the RVE

$S_{M}$ Compliance tensor of the matrix

$S_{F}$ Compliance tensor of a fiber

$\varphi_{F}$ Volume fraction of the fibers

$G_{M}$ Shear modulus of elasticity of the matrix

$\nu_{M}$ Poisson's ratio of the matrix

$H_{F}$ Compliance contribution tensor of all fibers in ${ }_{540}$ the RVE

$Q$ Hill's tensor

$q_{\alpha}$ Components of $Q$ expressed in standard tensor basis

$\kappa$ Coefficient defined in Eq. (30)

$f_{0}$ Coefficient defined in Eq. (31)

$f_{1}$ Coefficient defined in Eq. (32)

$\gamma$ Aspect ratio of an inhomogeneity

$g(\gamma)$ Shape function for an inhomogeneity with aspect ratio $\gamma$
Formulation: Below we present the formulation of this model.

$$
S_{e f f}=S_{M}+\left\{\left[H_{F}\right]^{-1}-Q\right\}^{-1}
$$

Where:

$$
H_{F}=\varphi_{F}\left[\left(S_{F}-S_{M}\right)^{-1}+Q\right]^{-1}
$$

The Hill's tensor for the fibers, $Q$, has the components $q_{\alpha}$, in standard tensor basis:

$$
\begin{gathered}
q_{1}=G_{M}\left[4 \kappa-1-2(3 \kappa-1) f_{0}-2 f_{1}\right] \\
q_{2}=2 G_{M}\left[1-(2-\kappa) f_{0}-f_{1}\right] \\
q_{3}=q_{4}=G_{M}\left[(2 \kappa-1) f_{0}+2 f_{1}\right] \\
q_{5}=4 G_{M}\left[f_{0}+4 f_{1}\right] \\
q_{6}=8 G_{M}\left[\kappa f_{0}-f_{1}\right]
\end{gathered}
$$

Given:

$$
\begin{gathered}
\kappa=\frac{1}{2\left(1-\nu_{M}\right)} \\
f_{0}=\frac{\gamma^{2}[1-g(\gamma)]}{2\left(\gamma^{2}-1\right)} ; \text { for cylinders, } f_{0}=\frac{1}{2} \\
f_{1}=\frac{\kappa \gamma^{2}}{4\left(\gamma^{2}-1\right)^{2}}\left[\left(2 \gamma^{2}+1\right) g(\gamma)-3\right] \\
\text { for cylinders, } f_{1}=0
\end{gathered}
$$

Additionally, the shape function $g(\gamma)$ is expressed in terms of the aspect ratio, $\gamma$, of the inhomogeneities. We assume that the inhomogeneities are fibers, and therefore are cylindrical and can be considered as prolate spheroids with an infinite aspect ratio $(\gamma \rightarrow$ $\infty)$. The general form of the shape function for prolate spheroids is:

$$
g(\gamma)=\frac{1}{2 \gamma \sqrt{\gamma^{2}-1}} \ln \frac{\gamma+\sqrt{\gamma^{2}-1}}{\gamma-\sqrt{\gamma^{2}-1}}
$$

\subsection{Chitin-Protein Nanofibrils}

The effective properties of a single chitin-protein nanofibril were determined using (1) a Mori-Tanaka model (Section 2.3.2) and (2) the ab-initio calculations of Nikolov et al. $(2011,2010)$ for the effective properties of $\alpha$-chitin and fiber proteins. We assume, based on previous research on beetles and other arthropods, that the $\alpha$-chitin crystals are wrapped in cylindrical proteins in a hexagonal arrangement and are of uniform diameter (Blackwell and Weih, 1980; Giraud-Guille et al., 1990; Neville et al., 1976). In some arthropod species, the resulting chitinprotein nanofibrils are tightly packed in a uniform, quasihexagonal arrangement to form a single chitin-protein microfiber, while in others, such as $C$. longinasus, the chitinprotein nanofibrils are embedded into a protein matrix 


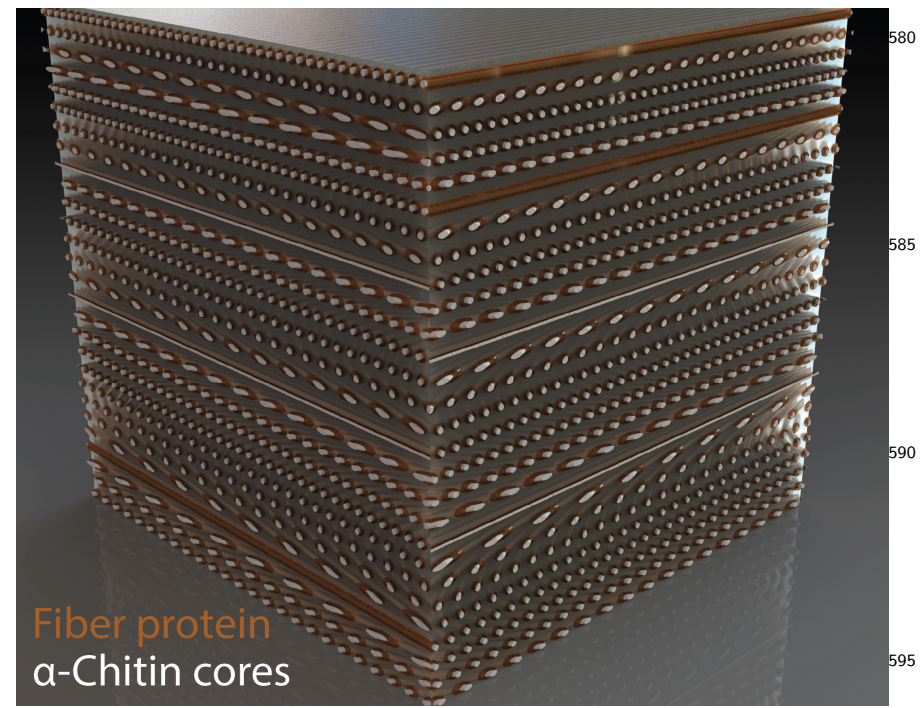

Figure 4: Interface of Bouligand structure between mesocuticle (bottom layers) and exocuticle (upper layers), showing gradual decrease in number of laminae per $180^{\circ}$ alternation (decreasing pitch) and a gradual increase in the number of chitin-protein nanofibrils per lamina (increasing volume fraction).

(Figs. 3 and 4) to form layers in the Bouligand structure (Blackwell and Weih, 1980; Chen et al., 2008; Cheng et al., 2008; Kaya et al., 2015; Raabe et al., 2005). For fibers embedded in a matrix with a hexagonal arrangement (Nikolov et al., 2010)

$$
\varphi_{F}=\frac{2 \pi r_{f}^{2}}{\sqrt{3}\left(2 r_{f}+s\right)^{2}}
$$

Because the microfibers are comprised solely of parallel nanofibrils, and given the assumptions of the Mori-Tanaka model, we may consider the effective properties of a homogenized microfiber and a homogenized nanofibril to be equivalent, physically differing only in radius. We therefore use data from Neville et al. (1976) for chitin-rich structures in beetles to calculate the volume fractions of chitin and protein from which we can derive, via the Mori-Tanaka model, the effective properties of a single chitin-protein nanofibril. We note that the values for the effective properties of the protein matrix come from relevant literature, as discussed below, and are different for the exocuticle, to reflect the increased cross-linking between the nanofibrils and the protein matrix that results from the process of tanning (sclerotization); also see Sections 3.1 and 3.3.

\subsection{Mesocuticle}

The effective properties of the helicoidal ply (Bouligand) structure of the mesocuticle can be ascertained in a600 three-step procedure that generally follows the method of Nikolov et al. (2011, 2010):

1. We first calculate the effective properties of a single, imperforate lamina by using the Mori-Tanaka method. We assume that a single ply (the RVE) in the Bouligand structure can be effectively modelled as a layer consisting of aligned, identical, evenly spaced, cylindrical inclusions (fibers) embedded in a matrix. As input, we take the effective stiffness tensor of a single chitin-protein nanofibril, the effective stiffness tensor of the protein matrix into which the nanofibrils are embedded, and the volume fraction of the nanofibrils, as calculated from Eq. (34). The exact separation distance between the nanofibrils is unknown, but it is possible to estimate this value based on the number of layers in the Bouligand structure, the size of the chitin-protein nanofibrils, and the pitch of the ply in our samples. We outline this method in greater numerical detail below (see Section 3.2).

2. Next, we simulate the helicoidal stacking of these layers to generate a transversely isotropic approximation of the Bouligand structure with a Voigt estimate (Section 2.3.1). At the macroscale, the Bouligand structure can be considered a transversely isotropic composite, so long as the number of plies is large (and can thus be considered infinitesimally thin) and the pitch of the ply (number of layers per $180^{\circ}$ rotation of the ply) is correspondingly small. Given the very large number of layers in the cuticle and relatively small pitch, the Bouligand structure can be considered transversely isotropic at small strains. We simulate this by considering $n$ layers (per $180^{\circ}$ alternation) stacked at $\theta$ degrees relative to each other along the normal axis, then taking a Voigt estimation (see Eq. (1)) over each layer where the properties of the $i^{\text {th }}$ layer are given by Reddy (2004):

$$
C_{i}=R: C: R^{T}
$$

Where $C$ is the stiffness tensor for a single lamina, and $R$ is given in terms of $\theta$ (Reddy, 2004):

$$
R=\left[\begin{array}{cccccc}
\cos ^{2} \theta & \sin ^{2} \theta & 0 & 0 & 0 & \sin 2 \theta \\
\sin ^{2} \theta & \cos ^{2} \theta & 0 & 0 & 0 & -\sin 2 \theta \\
0 & 0 & 1 & 0 & 0 & 0 \\
0 & 0 & 0 & \cos \theta & -\sin \theta & 0 \\
0 & 0 & 0 & \sin \theta & \cos \theta & 0 \\
-\sin \theta \cos \theta & \sin \theta \cos \theta & 0 & 0 & 0 & \cos ^{2} \theta-\sin ^{2} \theta
\end{array}\right]
$$

The Voigt estimate is therefore given, from Eqs. (1), (35), and (36):

$$
C=\frac{1}{n} \sum_{i=1}^{n} C_{i}
$$

3. Finally, we model the effect of adding pore canals to the structure. To calculate the effective properties of the helicoidal ply including pore canals, we utilize a non-interaction approximation (Section 2.3.3) from Sevostianov et al. (2005) rather than the threepoint approximation scheme of Hyun and Torquato (2000). We assume that the pores are cylindrical, rather than helico-ellipsoidal (Fig. 3), and have the same cross sectional area throughout. While sufficient for Nikolov et al. (2011, 2010), the three-point 
approximation scheme is not optimal for our purposes. Our scheme requires estimation of a complete effective stiffness tensor; yet the three point approximation only generates the in-plane elastic constants, not addressing the remaining constants. Sevostianov et al. (2005) found an explicit solution for calculating the effective properties of a material consisting of aligned spheroidal inclusions in a transversely isotropic matrix, when the axis of rotation of the inhomogeneities is aligned with the axis of transverse isotropy. For this reason we use their method and a non-interaction approximation to estimate the properties of the Bouligand structure with pores, based on the low volume fraction of pores in the cuticle of our samples, as explained below (see Section 3.2).

\subsection{Exocuticle}

The process for calculating the effective stiffness tensor of exocuticle is the same as that of mesocuticle, where we consider the material in three hierarchical levels: a single (imperforate) lamina, a laminate composite of stacked plies, and finally the Bouligand structure with pores. In our model, there are three main structural differences between the mesocuticle and exocuticle (see Section 3.3):

1. The packing of the laminae of the Bouligand structure is estimated to be approximately three times closer than mesocuticle, based on the pitch of the ply in exocuticle of our samples and data from other arthropods (Bouligand, 1972; Chen et al., 2008; Cheng et al., 2009). The reduced spacing also greatly increases the fiber fraction of the exocuticle so that the Mori-Tanaka method is no longer effective. To accommodate a high volume fraction of fibers and account for interactions between them, we utilize the effective field method (Section 2.3.4) for calculating the macroscale properties of the resulting individual ply (Sevostianov et al., 2005).

2. We estimate the effective Young's modulus of the fiber proteins to be significantly higher than in the endocuticle and mesocuticle, due to increases in crosslinking between the individual chitin-protein nanofibrils present in the exocuticle, as well as decreased water content. We use value presented by Vincent (1982), who provides measurements for the protein matrix of anatomical structures that exhibit large degrees of fiber cross-linking.

3. The effective Young's modulus of the protein matrix in which the nanofibrils are embedded is also estimated to be higher than in the mesocuticle, in keeping with the findings of Ker (1977), for highly sclerotized cuticle.

\subsection{Endocuticle}

Beetle endocuticle differs significantly in microstructural arrangement when compared to other layers of cuticle; namely, the endocuticle is characterized by the presence of unidirectional chitin macrofibers or balken (van de

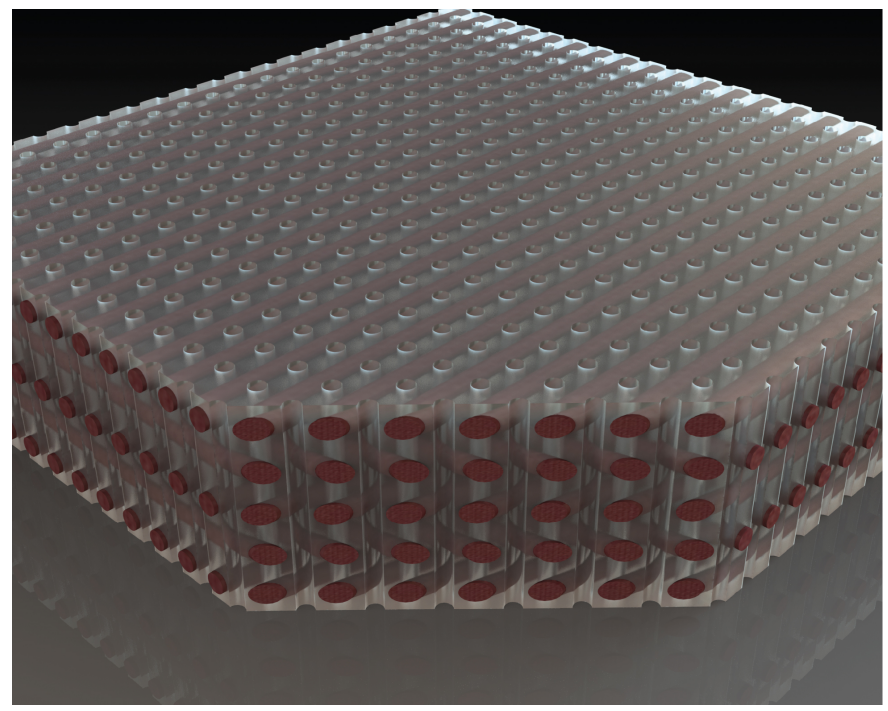

Figure 5: Typical "Balken-type" endocuticle, represented as orthogonally-stacked layers of chitin macrofibers embedded in a porous protein matrix; the pores exhibit a square arrangement between the macrofibers (not illustrated to scale).

Kamp and Greven, 2010). In these structures, the individual chitin cores surrounded by protein (the chitinprotein nanofibrils) are embedded in a protein matrix to form chitin-protein microfibers (Fig. 5). The latter, in turn, are tightly conjoined to form the macrofibers (van de Kamp and Greven, 2010; Kaya et al., 2015; Hepburn and Ball, 1973). The endocuticle generally exhibits the smallest degree of fiber cross-linking in the cuticle as a whole, and is the least sclerotized portion of this tissue (Vincent, 1982; Vincent and Wegst, 2004). We therefore adjust the properties of the protein matrices accordingly in our formulation of a micromechanical model for the endocuticle, as described in Section 3.4.

Among the Coleoptera, there are two main types of structural arrangements of the macrofibers that can occur (van de Kamp and Greven, 2010). The first type is considered the "classical" balken-type cuticle, where the macrofibers are arranged unidirectionally and are connected by a thin layer of protein matrix with interspersed pores. The second type is referred to as "reticulate" balkentype cuticle, which is characterized by cross-linking fibers between the balken that connect the macrofibers and also reinforce the pores. The latter type creates a linear mesh, where the gaps are filled by protein matrix and have central pores (Hepburn and Ball, 1973). In between these there are cuticles that exhibit only a small amount of crosslinking between macrofibers, and are very similar to the classical type; this was observed in Anthonomus grandis by Leopold et al. (1992). To simulate the micromechanical properties of a layer comprised of multiple balken, protein matrix, and pores, it is necessary to replicate the arrangement and volume fractions of these components within a RVE. We describe our general approach for endocuticle in 
four steps:
1. We first compute the effective properties of a single ${ }^{730}$ chitin macrofiber (our RVE at this step), given the properties of a homogenized chitin-protein nanofibril, the diameter and separation of the nanofibrils within the microfibers, and the properties of the protein matrix in which the nanofibrils are embedded. ${ }^{735}$ The lateral separation of the nanofibrils is known from recent work in weevils by van de Kamp et al. (2015) (see Section 3.4), thus leaving the effective properties of the matrix as the only unknown. We therefore take these properties from Nikolov et al. ${ }^{740}$ (2010). Although their data relate to the lobster endocuticle, we presume that their estimations are similar enough to be applicable the weevil endocuticle. This reasoning is supported both by the results outlined below and by findings for other in- ${ }^{745}$ sects (Ker, 1977; Vincent, 1982; Vincent and Wegst, 2004). Based on the volume fraction of microfibers observed in our samples, we utilize the effective field method to calculate the effective properties for a single, unidirectional macrofiber.

2. We assume, based on the observations of weevil balken by Leopold et al. (1992) and Davis (2014), that the even spacing of the macrofibers in our samples is due to a reduced amount of cross-linking between macrofibers, such that they can be considered effectively separated along most of their length by protein matrix. We may thus model a single balken layer755 by assuming that the overall architecture is that of a unidirectional, fiber-reinforced composite with a transversely isotropic fiber fraction and an isotropic matrix embedded with cylindrical pores. This enables us to calculate the volume fractions of the lay-760 ers of protein matrix, pores, and macrofibers, respectively, from the cross sectional areas of the respective elements of the structure and based on our samples. Furthermore, we assume a square arrangement of the pores due to the linear mesh arrangement of the balken layers, wherein:

$$
\varphi_{F}=\frac{2 \pi r_{f}^{2}}{\left(2 r_{f}+s\right)^{2}}
$$

Because the volume fraction of pores in our sample is quite low (see Section 3.4), we utilize a noninteraction approximation to homogenize the poreembedded matrix.

3. Our strategy then follows the approach of Vilchevskaya and Sevostianov (2015) for two-phase composites. We do not attempt to simultaneously calculate the combined effect of pores and fibers, which are assumed to be cylindrical, aligned, identical (within each respective phase, pore or fiber), and orthogonal to each other in the matrix, since it is possible that the combined effect of a stiff phase and a compliant phase can lead to numerical singularity at cer- tain volume fractions (Sevostianov, 2014). Rather, we first calculate the effective properties of the poreembedded matrix, and then create an isotropic approximation of the resulting transversely isotropic, homogenized material. Because the resulting degree of transverse isotropy is correspondingly low, an isotropic approximation (Section 2.3.5) can be used with reasonable accuracy to calculate the Hill's tensor for the macrofibers ${ }^{3}$, as explained below.

4. Given an isotropic approximation for the protein matrix embedded with cylindrical pores, we can now simulate the effect of adding macrofibers to the (porous) matrix via a Maxwell homogenization scheme (Section 2.3.6). The isotropic approximation is used to calculate the Hill's tensor for the macrofibers, $Q$, used in Eq. (28), and whose components are given in Eqs. (29). We assume that the interaction between pores and the fiber fraction is essentially negligible, though we utilize the compliance tensor of the transversely isotropic, pore-embedded matrix, rather than the isotropic approximation, to calculate the effective compliance tensor of the balken layer in Eq. (27).

\section{Results}

In this section, we examine the explicit numerical solutions of the models as outlined in the preceding section. We begin at the lowest hierarchical level for each region of cuticle, building up to the complete structure sequentially, at each level in the structural hierarchy. We also report, where appropriate, values obtained from relevant literature for certain parameters that we were not able to measure experimentally. With this foundation, we are able to present the macroscale properties of each layer of the cuticle, as well as examine the robustness of the resultant elastic constants with respect to literature-extracted model parameters.

\subsection{Chitin-Protein Nanofibrils}

Nikolov et al. $(2011,2010)$ specify the stiffness tensor for crystalline $\alpha$-chitin, with the longitudinal axis of the fiber aligned with axis 1 , in $6 \times 6$ matrix notation configured as:

$$
C_{C H}=\left[\begin{array}{cccccc}
119 & 0.10 & 1.1 & 0 & 0 & 0 \\
0.10 & 28 & 2.0 & 0 & 0 & 0 \\
1.1 & 2.0 & 24 & 0 & 0 & 0 \\
0 & 0 & 0 & 5.0 & 0 & 0 \\
0 & 0 & 0 & 0 & 8.0 & 0 \\
0 & 0 & 0 & 0 & 0 & 2.0
\end{array}\right]
$$

Assuming that the fiber protein matrix is isotropic and that Young's modulus and Poisson's ratio are $E_{F P}=0.004$

\footnotetext{
${ }^{3}$ This crucial step enables us to approximate the effective properties of this two-phase composite, while simultaneously sidestepping two particular complications: potential numerical singularity during tensor inversion (Sevostianov, 2014), and the problem of explicitly calculating the effective properties of fibers embedded in a transversely isotropic matrix, where the fibers are aligned orthogonal to the axis of transverse isotropy (Saadat et al., 2012; Vilchevskaya and Sevostianov, 2015).
} 
GPa and $\nu_{F P}=0.49$, respectively Nikolov et al. (2011, 2010), and given the general form of a stiffness tensor for isotropic media from Eq. (26), the stiffness tensor of the fiber proteins is specified as:

$$
C_{F P}=\left[\begin{array}{cccccc}
0.068 & 0.066 & 0.066 & 0 & 0 & 0 \\
0.066 & 0.068 & 0.066 & 0 & 0 & 0 \\
0.066 & 0.066 & 0.068 & 0 & 0 & 0 \\
0 & 0 & 0 & 0.0013 & 0 & 0 \\
0 & 0 & 0 & 0 & 0.0013 & 0 \\
0 & 0 & 0 & 0 & 0 & 0.0013
\end{array}\right]
$$

In Coleoptera belonging to two different families (Scarabaeidae and Tenebrionidae), the $\alpha$-chitin crystallites in chitinrich structures show a quasi-hexagonal arrangement Neville et al. (1976). The diameter of individual $\alpha$-chitin crystallites have been measured, on average, as $2 r_{f}=2.71,2.86 \mathrm{~nm}$ with the center to center distances as $5.9,5.3 \mathrm{~nm}$ respectively, and translating into lateral separation distances of $s=3.19,2.44 \mathrm{~nm}$, respectively Neville et al. (1976). Nikolov et al. Nikolov et al. (2011) reported that these values can vary without noticeable influence on the resultant macro-scale properties of the cuticle. Hence we apply averages of these measures such that $2 r_{f}=2.785 \mathrm{~nm}$ and $s=2.815 \mathrm{~nm}$, and consequently (given Eq. [34]), $\varphi_{C H}=$ 0.22 and $\varphi_{F P}=0.78$. Because the volume fraction is less than 30\%, we use the Mori-Tanaka model described in Section 2.3.2.

The Eshelby's tensor for the $\alpha$-chitin crystallites is dependent only on Poisson's ratio of the matrix $\left(\nu_{F P}=\right.$ 0.49), and can be found by Eqs. (4) and (5):

$$
S_{C H}=\left[\begin{array}{cccccc}
0 & 0 & 0 & 0 & 0 & 0 \\
0.45 & 0.75 & 0.24 & 0 & 0 & 0 \\
0.45 & 0.24 & 0.75 & 0 & 0 & 0 \\
0 & 0 & 0 & 0.51 & 0 & 0 \\
0 & 0 & 0 & 0 & 0.50 & 0 \\
0 & 0 & 0 & 0 & 0 & 0.50
\end{array}\right]
$$

We can thus calculate the strain concentration factor from Eq. (3):

$$
A_{C H}=\left[\begin{array}{cccccc}
1.0 & 0 & 0 & 0 & 0 & 0 \\
0.0019 & 0.0024 & 0.0022 & 0 & 0 & 0 \\
-0.043 & 0.0026 & 0.0028 & 0 & 0 & 0 \\
0 & 0 & 0 & 0.00053 & 0 & 0 \\
0 & 0 & 0 & 0 & 0.00034 & 0 \\
0 & 0 & 0 & 0 & 0 & 0.0013
\end{array}\right]
$$

Given Eq. (2), the stiffness tensor of a chitin protein nanofibril is specified as:

$$
C_{N F}=\left[\begin{array}{cccccc}
27 & 0.067 & 0.067 & 0 & 0 & 0 \\
0.067 & 0.089 & 0.085 & 0 & 0 & 0 \\
0.067 & 0.085 & 0.089 & 0 & 0 & 0 \\
0 & 0 & 0 & 0.0021 & 0 & 0 \\
0 & 0 & 0 & 0 & 0.0021 & 0 \\
0 & 0 & 0 & 0 & 0 & 0.0021
\end{array}\right]
$$

\subsection{Mesocuticle}

Here we present the numerical results of the four-step procedure outlined in Section 2.5.

1. To calculate the effective properties of a single layer of the Bouligand structure as a fiber-reinforced matrix, we need to account for the stiffness tensor of the chitin-protein nanofibrils and protein matrix, as well as their respective volume fractions. The stiffness tensor of the chitin-protein nanofibrils is given by Eq. (43). The volume fractions may be calculated from Eq. (34), assuming a quasi-hexagonal arrangement of the fibers. The exact separation between the nanofibrils is unknown, but it is possible to estimate their separation based on the number of layers in the Bouligand structure, the size of the chitin-protein nanofibrils, and the pitch of the ply. In our samples, there are (on average) approximately ten $180^{\circ}$ alternations per $1 \mu \mathrm{m}$. We therefore estimate approximately $100 \mathrm{~nm}$ per $180^{\circ}$ alternation. The nanofibrils have an estimated diameter of $5.6 \mathrm{~nm}$ each, based on Section 3.1. If we assume a separation distance of 1.5 times the radius of a nanofibril $(s=4.2 \mathrm{~nm})$, the resulting structure has roughly 10 layers per $180^{\circ}$ alternation. This number agrees with experimentally measured stacking in the mesocuticle of beetles (Cheng et al., 2009). Moreover, these parameters give the model macroscale properties that show very good correspondence with fresh mesocuticle (Klocke and Schmitz, 2011).

The above specifications yield $\varphi_{N F}=0.30$ as an upper bound for the volumetric fraction of nanofibrils in the apparent mesocuticle and therefore $\varphi_{P M}=$ 0.70 . The volume fraction of chitin is therefore nearly $6.5 \%$, which agrees with experimentally measured values of chitin inclusion (Vincent, 1982; Vincent and Wegst, 2004). Given this result, the Mori-Tanaka model may be used to find the effective stiffness tensor of a single ply within the Bouligand structure of the apparent mesocuticle, without pore canals. This volume fraction is generally considered to be at the upper limit (30\%) for this method; in this instance, however, we find that estimation of the effective tensor for mesocuticle using the Maxwell scheme yields nearly identical results.

Young's moduli, $E_{P M}$, for matrix proteins have been reported by Ker (1977) to vary from $0.12 \mathrm{GPa}$ in tendons to $1.7 \mathrm{GPa}$ in the highly sclerotized tissue (tibia). Poisson's ratio, $\nu_{P M}$, of these proteins is unknown for insects, but was found by Nikolov et al. (2011) to be 0.45 for lobsters. To calculate the stiffness tensor of the protein matrix, we assume $E_{P M}=$ $1.2 \mathrm{GPa}$ and $\nu_{P M}=0.45$ because of the degree of tanning and sclerotization in the mesocuticle. We therefore specify:

$$
C_{P M}=\left[\begin{array}{cccccc}
4.6 & 3.7 & 3.7 & 0 & 0 & 0 \\
3.7 & 4.6 & 3.7 & 0 & 0 & 0 \\
3.7 & 3.7 & 4.6 & 0 & 0 & 0 \\
0 & 0 & 0 & 0.41 & 0 & 0 \\
0 & 0 & 0 & 0 & 0.41 & 0 \\
0 & 0 & 0 & 0 & 0 & 0.41
\end{array}\right]
$$

Accordingly, Eshelby's tensor for a chitin-protein nanofibril is:

$$
S_{N F}=\left[\begin{array}{cccccc}
0 & 0 & 0 & 0 & 0 & 0 \\
0.41 & 0.73 & 0.18 & 0 & 0 & 0 \\
0.41 & 0.18 & 0.73 & 0 & 0 & 0 \\
0 & 0 & 0 & 0.55 & 0 & 0 \\
0 & 0 & 0 & 0 & 0.50 & 0 \\
0 & 0 & 0 & 0 & 0 & 0.50
\end{array}\right]
$$


The strain concentration factor for the nanofiobrils is modeled as:

$$
A_{N F}=\left[\begin{array}{cccccc}
1.0 & 0 & 0 & 0 & 0 & 0 \\
3.7 & 5.6 & 3.5 & 0 & 0 & 0 \\
3.7 & 3.5 & 5.6 & 0 & 0 & 0 \\
0 & 0 & 0 & 2.2 & 0 & 0 \\
0 & 0 & 0 & 0 & 2.0 & 0 \\
0 & 0 & 0 & 0 & 0 & 2.0
\end{array}\right]
$$

Lastly, the stiffness tensor of a single lamina of the Bouligand structure is:

$$
C_{L}=\left[\begin{array}{cccccc}
9.5 & 0.82 & 0.82 & 0 & 0 & 0 \\
0.82 & 1.1 & 0.71 & 0 & 0 & 0 \\
0.82 & 0.71 & 1.1 & 0 & 0 & 0 \\
0 & 0 & 0 & 0.22 & 0 & 0 \\
0 & 0 & 0 & 0 & 0.23 & 0 \\
0 & 0 & 0 & 0 & 0 & 0.23
\end{array}\right]
$$

2. A Voigt estimate (Section 2.3.1) can be applied to find the effective upper bound of the stiffness tensor of the entire Bouligand structure according to Eqs. (35)-(37). The rotation matrix is necessary to find the effective properties of all three plies in the same coordinate system, as they are rotated relative to each other. Axis 3 is used as the transverse normal to the laminae, while axes 1 and 2 are the in-plane laminar axes. The simplest laminate composite conforming to the generalized Bouligand structure is a stack of three laminae each rotated $60^{\circ}$ to each other along the normal axis. We therefore assume that $i=1$ represents the instance of the lamina whose properties are already represented in the appropriate coordinate system, where $\theta=0^{\circ}$, and given in Eq. (47). Thus, given that $\theta=60^{\circ}, 120^{\circ}$, when $i=2,3$, respectively:

$$
\begin{gathered}
C_{1}=C_{L} \\
C_{2}=\left[\begin{array}{cccccc}
1.7 & 2.3 & 0.80 & 0 & 0 & 0.93 \\
2.3 & 5.9 & 0.80 & 0 & 0 & 2.7 \\
0.80 & 0.80 & 1.1 & 0 & 0 & 0.050 \\
0 & 0 & 0 & 0.22 & 0.0042 & 0 \\
0 & 0 & 0 & 0.0042 & 0.22 & 0 \\
0.93 & 2.7 & 0.050 & 0 & 0 & 1.7
\end{array}\right] \\
C_{3}=\left[\begin{array}{cccccc}
1.7 & 2.3 & 0.80 & 0 & 0 & -0.93 \\
2.3 & 5.9 & 0.80 & 0 & 0 & -2.7 \\
0.80 & 0.80 & 1.1 & 0 & 0 & -0.050 \\
0 & 0 & 0 & 0.22 & -0.0042 & 0 \\
0 & 0 & 0 & -0.0042 & 0.22 & 0 \\
-0.93 & -2.7 & -0.050 & 0 & 0 & 1.7
\end{array}\right]
\end{gathered}
$$

Therefore, for the Bouligand structure without pores ${ }^{4}$ :

$$
C_{P W}=\left[\begin{array}{cccccc}
4.3 & 1.8 & 0.77 & 0 & 0 & 0 \\
1.8 & 4.3 & 0.77 & 0 & 0 & 0 \\
0.77 & 0.77 & 1.1 & 0 & 0 & 0 \\
0 & 0 & 0 & 0.22 & 0 & 0 \\
0 & 0 & 0 & 0 & 0.22 & 0 \\
0 & 0 & 0 & 0 & 0 & 1.2
\end{array}\right]
$$

3. Sevostianov et al. (2005) calculated the effective properties of a transversely isotropic matrix embedded with spheroidal inhomogeneities whose rotational axes are aligned with the axis of symmetry in the matrix. If the Bouligand structure can be effectively represented as a transversely isotropic matrix, and the pore canals as cylinders, rather than helices, then

\footnotetext{
${ }^{4}$ This result is the accurate to at least twelve decimal places re-800 gardless of the number of laminae used.
}

we can calculate the additional compliance added to the material by an arbitrary number of pores. In general, this is as two-step procedure: first, the additional compliance imbued by a single pore must be ascertained, then the influence of multiple pores can be calculated, based on the shape and number of pores.

Unlike the cuticle of other arthropods, such as lobsters, beetle cuticle does not have an extensively developed pore canal system, and often appears in SEM or TEM to have a solid surface area, $\varphi_{S}$, in excess of $\varphi_{S}=0.96$ ((Cheng et al., 2009; Hepburn and Ball, 1973; Hyun and Torquato, 2000; McNamara et al., 2012)). In keeping with their findings, we assume a pore fraction of $\varphi_{P C}=0.04$ as an upper bound, although the exact area fraction of the pores is not known for any member of the genus Curculio, and is quite possibly much lower. Because the pore canal fraction is very low, we can assume that the interaction between pore canals is essentially negligible. We therefore use a non-interaction approximation, described in Section 2.3.3, to simulate the effect of introducting pore canals into the Bouligand structure (Kachanov and Sevostianov, 2005; Sevostianov and Kachanov, 2002; Sevostianov et al., 2005).

We begin by calculating the compliance tensor of the stacked laminae, $S_{P W}$, from the stiffness tensor presented in Eq. (49):

$$
S_{P W}=\left[C_{P W}\right]^{-1}=\left[\begin{array}{cccccc}
0.30 & -0.10 & -0.13 & 0 & 0 & 0 \\
-0.10 & 0.30 & -0.13 & 0 & 0 & 0 \\
-0.13 & -0.13 & 1.1 & 0 & 0 & 0 \\
0 & 0 & 0 & 4.5 & 0 & 0 \\
0 & 0 & 0 & 0 & 4.5 & 0 \\
0 & 0 & 0 & 0 & 0 & 0.81
\end{array}\right]
$$

From Eq. (11)-(16), we derive:

$$
H_{N I}=\left[\begin{array}{cccccc}
0.027 & -0.019 & -0.0053 & 0 & 0 & 0 \\
-0.019 & 0.027 & -0.0053 & 0 & 0 & 0 \\
-0.0053 & -0.0053 & 0.042 & 0 & 0 & 0 \\
0 & 0 & 0 & 0.090 & 0 & 0 \\
0 & 0 & 0 & 0 & 0.090 & 0 \\
0 & 0 & 0 & 0 & 0 & 0.023
\end{array}\right]
$$

Therefore, given Eq. (6), the compliance tensor for mesocuticle is:

$$
S_{M}=\left[\begin{array}{cccccc}
0.32 & -0.12 & -0.14 & 0 & 0 & 0 \\
-0.12 & 0.32 & -0.14 & 0 & 0 & 0 \\
-0.14 & -0.14 & 1.1 & 0 & 0 & 0 \\
0 & 0 & 0 & 4.6 & 0 & 0 \\
0 & 0 & 0 & 0 & 4.6 & 0 \\
0 & 0 & 0 & 0 & 0 & 0.83
\end{array}\right]
$$

And, the stiffness tensor for mesocuticle is specified as:

$$
C_{M}=\left[\begin{array}{cccccc}
4.1 & 1.8 & 0.74 & 0 & 0 & 0 \\
1.8 & 4.1 & 0.74 & 0 & 0 & 0 \\
0.74 & 0.74 & 1.1 & 0 & 0 & 0 \\
0 & 0 & 0 & 0.22 & 0 & 0 \\
0 & 0 & 0 & 0 & 0.22 & 0 \\
0 & 0 & 0 & 0 & 0 & 1.2
\end{array}\right]
$$

\subsection{Exocuticle}

In our model, we identify three primary differences between mesocuticle and exocuticle (see also Section 2.6): 
1. The packing of the laminae is estimated to be approximately 2-3 times closer than mesocuticle based on the pitch of the ply in exocuticle of our samples, and data from other arthropods (Bouligand, 1972; Chen et al., 2008; Cheng et al., 2009; Klocke and Schmitz, 2011). We posit that the diameter of the $\mathrm{e}_{835}$ nanofibrils remains the same, but that the pitch of the ply and separation distance between nanofibrils are reduced. Assuming a lateral separation equal to one-quarter of the radius $(s=0.7 \mathrm{~nm})$ and half as many laminae per $180^{\circ}$ alternation, the resulting structure has three times as many alternations as mesocuticle. The reduced spacing also greatly increases the fiber fraction of the exocuticle, such that $\varphi_{N F}=0.72$ (total volume of chitin rises to $16.1 \%$ ). This volume fraction violates the assumption of dilute inclusions used by the Mori-Tanaka model, and we therefore use the effective field method presented in Sevostianov et al. (2005) to derive the effective properties of a single lamina of the exocuticle.

2. We estimate the effective Young's modulus of the fiber proteins as $E_{F P}=0.12 \mathrm{GPa}$, on the basis of Vincent (1982), who provides measurements for the protein matrix of anatomical structures that exhibit large degrees of fiber cross-linking. Changing this parameter of the Mori-Tanaka model presented in Section 3.1 yields:

$$
C_{N F}=\left[\begin{array}{cccccc}
28 & 1.9 & 1.9 & 0 & 0 & 0 \\
1.9 & 2.6 & 2.4 & 0 & 0 & 0 \\
1.9 & 2.4 & 2.6 & 0 & 0 & 0 \\
0 & 0 & 0 & 0.063 & 0 & 0 \\
0 & 0 & 0 & 0 & 0.063 & 0 \\
0 & 0 & 0 & 0 & 0 & 0.062
\end{array}\right]
$$

3. The effective Young's modulus of the protein matrix in which the microfibers are embedded is estimated as $E_{P M}=1.7 \mathrm{GPa}$, in keeping with the findings of Ker Ker (1977), for highly sclerotized cuticle.

To use the effective field method, it is necessary to first transform the tensors used as input so that the axis of rotation of an inclusion and the axis of transverse isotropy of the matrix are aligned with the global axis 3 . The stiffness tensor presented above has the longitudinal axis of the fiber aligned with global axis 1 . The transformed matrix representation can be specified as:

$$
C_{N F}=\left[\begin{array}{cccccc}
2.6 & 2.4 & 1.9 & 0 & 0 & 0 \\
2.4 & 2.6 & 1.9 & 0 & 0 & 0 \\
1.9 & 1.9 & 28 & 0 & 0 & 0 \\
0 & 0 & 0 & 0.062 & 0 & 0 \\
0 & 0 & 0 & 0 & 0.063 & 0 \\
0 & 0 & 0 & 0 & 0 & 0.063
\end{array}\right]
$$

The protein matrix is isotropic and does not need to be rotated; however, given the higher Young's modulus of the matrix, we determine:

$$
C_{P M}=\left[\begin{array}{cccccc}
6.4 & 5.3 & 5.3 & 0 & 0 & 0 \\
5.3 & 6.4 & 5.3 & 0 & 0 & 0 \\
5.3 & 5.3 & 6.4 & 0 & 0 & 0 \\
0 & 0 & 0 & 0.59 & 0 & 0 \\
0 & 0 & 0 & 0 & 0.59 & 0 \\
0 & 0 & 0 & 0 & 0 & 0.59
\end{array}\right]
$$

And, from Eq. (21):

$$
C_{D}=\left[\begin{array}{cccccc}
-3.9 & -2.8 & -3.4 & 0 & 0 & 0 \\
-2.8 & -3.9 & -3.4 & 0 & 0 & 0 \\
-3.4 & -3.4 & 22 & 0 & 0 & 0 \\
0 & 0 & 0 & -0.52 & 0 & 0 \\
0 & 0 & 0 & 0 & -0.52 & 0 \\
0 & 0 & 0 & 0 & 0 & -0.52
\end{array}\right]
$$

Therefore, for a single lamina we can specify (following rotation back to the original coordinate system $)^{5}$ :

$$
C_{L}=\left[\begin{array}{cccccc}
22 & 2.5 & 2.5 & 0 & 0 & 0 \\
2.5 & 3.2 & 2.9 & 0 & 0 & 0 \\
2.5 & 2.9 & 3.2 & 0 & 0 & 0 \\
0 & 0 & 0 & 0.15 & 0 & 0 \\
0 & 0 & 0 & 0 & 0.16 & 0 \\
0 & 0 & 0 & 0 & 0 & 0.16
\end{array}\right]
$$

For the Bouligand structure (using a Voigt estimate):

$$
C_{P W}=\left[\begin{array}{ccccccc}
10 & 4.8 & 2.7 & 0 & 0 & 0 \\
4.8 & 10 & 2.7 & 0 & 0 & 0 \\
2.7 & 2.7 & 3.2 & 0 & 0 & 0 \\
0 & 0 & 0 & 0.15 & 0 & 0 \\
0 & 0 & 0 & 0 & 0.15 & 0 \\
0 & 0 & 0 & 0 & 0 & 2.6
\end{array}\right]
$$

Assuming the same porosity as the mesocuticle, the effective tensor for the exocuticle is:

$$
C_{X}=\left[\begin{array}{cccccc}
9.5 & 4.7 & 2.6 & 0 & 0 & 0 \\
4.7 & 9.5 & 2.6 & 0 & 0 & 0 \\
2.6 & 2.6 & 3.1 & 0 & 0 & 0 \\
0 & 0 & 0 & 0.15 & 0 & 0 \\
0 & 0 & 0 & 0 & 0.15 & 0 \\
0 & 0 & 0 & 0 & 0 & 2.5
\end{array}\right]
$$

\subsection{Endocuticle}

Here we present the results of the general approach outlined for the derivation of the effective properties of endocuticle, as outlined in Section 2.7:

1. Beetle endocuticle is characterized by the presence of unidirectional chitin macrofibers (van de Kamp and Greven, 2010). In these structures, the individual chitin cores surrounded by protein (the chitinprotein nanofibrils) form chitin-protein microfibers that can vary in size from $20-90 \mathrm{~nm}$ in diameter (Kaya et al., 2015). These microfibers are tightly conjoined to form the macrofibers. Hepburn and Ball (1973) found the approximate size of microfibers in Pachynoda sinuata (Fabricius 1775) to be approximately $30 \mathrm{~nm}$. This is close to the diameter and separation of fibers that can be seen in Anthonomus grandis Boheman 1843 (Leopold et al., 1992). In our samples, we observed that these fibers are of a similar size on average. Because these microfibers appear to be tightly bound, and are not visibly separated in transmission electron micrographs [see van de Kamp et al. (2015)], we treat the macrofibers as if they are solely comprised of aligned nanofibrils embedded in a matrix.

\footnotetext{
${ }^{5}$ It is worth noting that the formulation of the effective field method presented herein is for transversely isotropic fibers, rather than fully orthotropic ones. We treat the nanofibrils as transversely isotropic; however, reformulating to allow for orthotropic fibers does change the resultant stiffness tensor very slightly. Because these numerical differences are beyond the ninth decimal place for the values used herein, and essentially disappear with the Voigt estimate, we feel that this is not a critical factor in the calculation of the effective properties of the exocuticle.
} 
We can readily calculate the effective tensor for a single macrofiber in a manner similar to calculating the effective properties of a single ply in the exocuticle or mesocuticle, without canals. If we assume that the nanofibrils are separated by a distance equal to their own radius [as seen in van de Kamp et al. (2015)] in a quasi-hexagonal arrangement, we find, given Eq. (34), $\varphi_{F}=0.40$. We also assume $E_{F P}=0.004 \mathrm{GPa}$, to reflect the reduced crosslinking in the endocuticle, and that $E_{P M}=1.0 \mathrm{GPa}$, in keeping with the value used for endocuticle by Nikolov et al. (2010). With these values, we find the stiffness tensor for a the protein matrix as:

$$
C_{P M}=\left[\begin{array}{ccccccc}
3.8 & 3.1 & 3.1 & 0 & 0 & 0 \\
3.1 & 3.8 & 3.1 & 0 & 0 & 0 \\
3.1 & 3.1 & 3.8 & 0 & 0 & 0 \\
0 & 0 & 0 & 0.34 & 0 & 0 \\
0 & 0 & 0 & 0 & 0.34 & 0 \\
0 & 0 & 0 & 0 & 0 & 0.34
\end{array}\right]
$$

And the effective stiffness tensor for a single chitin macrofiber:

$$
C_{M F}=\left[\begin{array}{cccccc}
12 & 0.50 & 0.50 & 0 & 0 & 0 \\
0.50 & 0.71 & 0.43 & 0 & 0 & 0 \\
0.50 & 0.43 & 0.71 & 0 & 0 & 0 \\
0 & 0 & 0 & 0.14 & 0 & 0 \\
0 & 0 & 0 & 0 & 0.15 & 0 \\
0 & 0 & 0 & 0 & 0 & 0.15
\end{array}\right]
$$

2. In order to calculate the properties of a single balken layer, we posit that the layer is comprised of macrofibers, with effective stiffness $C_{M F}$, aligned in an isotropic protein matrix with stiffness identical to $C_{P M}$. In addition we assume that the layer is porous, and that the pores are embedded in the protein matrix between the macrofibers. From our samples, we find that the area fraction (and, equivalently, the volume fraction) of protein matrix layers varies, by layer, from roughly $14-18 \%$. We therefore take the average and set $\varphi_{L}=0.16$ and $\varphi_{M F}=0.84$. Additionally, the ratio of the diameter of a pore and its separation distance to the closest pore is equal to the area fraction of the protein layers in cross-section. We assume a square arrangement of the pores due to the linear mesh arrangement of the balken layers; thus, given Eq. (38), we find $\varphi_{p} \approx 0.015$.

We now consider an isotropic protein matrix with effective properties equal to $C_{P M}$. We assume a global coordinate system where axis 1 is the long axis of the macrofibers, axis 2 is the in-plane axis that, along with axis 1 , defines the plane of a single layer of balken, and axis 3 is the cuticle normal. We then introduce into this matrix a system of identical, cylindrical pores with the same arrangement of our samples, whose long axis is aligned with global axis 3. Because the pores in our samples are effectively embedded in the protein matrix between the macrofibers and occupy a very small volume, we can estimate the properties of the homogenized matrix, before introducing the macrofibers, using a noninteraction approximation (Section 2.3.3).
From the non-interaction approximation outlined in Section 2.3.3, we derive the following:

$$
H_{N I}=\left[\begin{array}{cccccc}
0.031 & -0.017 & -0.0067 & 0 & 0 & 0 \\
-0.017 & 0.031 & -0.0067 & 0 & 0 & 0 \\
-0.0067 & -0.0067 & 0.015 & 0 & 0 & 0 \\
0 & 0 & 0 & 0.022 & 0 & 0 \\
0 & 0 & 0 & 0 & 0.022 & 0 \\
0 & 0 & 0 & 0 & 0 & 0.028
\end{array}\right]
$$

Thus, given $C_{P M}$ and Eq. (6), the effective compliance tensor for a layer of protein separating the macrofibers is obtained as:particular sets of factors

$$
S_{L}=\left[\begin{array}{cccccc}
1.0 & -0.47 & -0.46 & 0 & 0 & 0 \\
-0.47 & 1.0 & -0.46 & 0 & 0 & 0 \\
-0.46 & -0.46 & 1.0 & 0 & 0 & 0 \\
0 & 0 & 0 & 2.9 & 0 & 0 \\
0 & 0 & 0 & 0 & 2.9 & 0 \\
0 & 0 & 0 & 0 & 0 & 2.9
\end{array}\right]
$$

And, therefore:

$$
C_{L}=\left[\begin{array}{cccccc}
3.6 & 2.9 & 3.0 & 0 & 0 & 0 \\
2.9 & 3.6 & 3.0 & 0 & 0 & 0 \\
3.0 & 3.0 & 3.6 & 0 & 0 & 0 \\
0 & 0 & 0 & 0.34 & 0 & 0 \\
0 & 0 & 0 & 0 & 0.34 & 0 \\
0 & 0 & 0 & 0 & 0 & 0.34
\end{array}\right]
$$

3. Because the pores occupy a small volume, the anisotropic generated by their inclusion will be relatively small. We are therefore able to generate an isotropic approximation of the matrix to calculate the stiffness contribution tensor $N_{M F}$ and Hill's tensor $Q_{M F}$ for a macrofiber. Considering a transversely isotropic layer of porous protein matrix, with stiffness tensor $C_{L}$, the isotropic approximation of the shear modulus $G$ and bulk modulus $K$, as well as Young's modulus $E$ and Poisson's ratio $\nu$ are given by Eqs. (24) and (25), respectively:

$$
\begin{gathered}
G=0.34 \mathrm{GPa} \\
K=3.2 \mathrm{GPa} \\
E=0.98 \mathrm{GPa} \\
\nu=0.45
\end{gathered}
$$

From these elastic constants, and through application of Eq. (23), we can calculate the isotropic approximations of the effective stiffness and compliance tensors of the protein matrix:

$$
S_{I L}=\left[\begin{array}{cccccc}
1.0 & -0.46 & -0.46 & 0 & 0 & 0 \\
-0.46 & 1.0 & -0.46 & 0 & 0 & 0 \\
-0.46 & -0.46 & 1.0 & 0 & 0 & 0 \\
0 & 0 & 0 & 2.9 & 0 & 0 \\
0 & 0 & 0 & 0 & 2.9 & 0 \\
0 & 0 & 0 & 0 & 0 & 2.9
\end{array}\right]
$$

4. Using the isotropic approximation of the porous matrix, we now calculate the effective properties of a single balken layer by simulating the effect of embedding macrofibers into the matrix, using the Maxwell homogenization scheme described in Section 2.3.6. Given the elastic constants in Eq. (66) and the components of Hill's tensor, Q, presented in Eqs. (29), 
we obtain ${ }^{6}$ :

$$
Q_{M F}=\left[\begin{array}{cccccc}
1.2 & 0.28 & 0.28 & 0 & 0 & 0 \\
0.28 & 0.46 & 0.15 & 0 & 0 & 0 \\
0.28 & 0.15 & 0.46 & 0 & 0 & 0 \\
0 & 0 & 0 & 0.15 & 0 & 0 \\
0 & 0 & 0 & 0 & 0.17 & 0 \\
0 & 0 & 0 & 0 & 0 & 0.17
\end{array}\right]
$$

Thus, given Eq. (28), the compliance contribution tensor for the macrofibers is:

$$
H_{M F}=\left[\begin{array}{cccccc}
-9.0 & 4.0 & 4.0 & 0 & 0 & 0 \\
4.0 & -1.1 & -2.1 & 0 & 0 & 0 \\
4.0 & -2.1 & -1.1 & 0 & 0 & 0 \\
0 & 0 & 0 & 2.1 & 0 & 0 \\
0 & 0 & 0 & 0 & 1.9 & 0 \\
0 & 0 & 0 & 0 & 0 & 1.9
\end{array}\right]
$$

Using Eq. (27), the compliance stiffness tensors for a single balken layer are, respectively:

$$
\begin{gathered}
S_{B}=\left[\begin{array}{cccccc}
0.12 & -0.057 & -0.047 & 0 & 0 & 0 \\
-0.057 & 1.9 & -1.2 & 0 & 0 & 0 \\
-0.047 & -1.2 & 1.9 & 0 & 0 & 0 \\
0 & 0 & 0 & 6.1 & 0 & 0 \\
0 & 0 & 0 & 0 & 5.8 & 0 \\
0 & 0 & 0 & 0 & 0 & 5.8
\end{array}\right] \\
C_{B}=\left[\begin{array}{ccccccc}
9.0 & 0.63 & 0.60 & 0 & 0 & 0 \\
0.63 & 0.86 & 0.53 & 0 & 0 & 0 \\
0.60 & 0.53 & 0.86 & 0 & 0 & 0 \\
0 & 0 & 0 & 0.16 & 0 & 0 \\
0 & 0 & 0 & 0 & 0.17 & 0 \\
0 & 0 & 0 & 0 & 0 & 0.17
\end{array}\right]
\end{gathered}
$$

\subsection{Summary of Results}

In Eq. (72) [see following page] we present the effective stiffness tensors (in $6 \times 6$ matrix notation) for the exocuticle, mesocuticle, and a single balken layer of the ${ }^{875}$ endocuticle, as $C_{X}, C_{M}$, and $C_{B}$, respectively. Additionally, we list the elastic constants that can be calculated from the inverse of these tensors in Tab. 1 and Fig. 6, and summarize the models from which these tensors were derived in Tab. 2. Accordingly, the in-plane (axes 1 and 2) (280 $^{2}$ and $3.08 \mathrm{GPa}$, respectively, while the transverse (axis 3 ) moduli for these regions are $2.14 \mathrm{GPa}$ and $0.91 \mathrm{GPa}$, respectively. Interestingly, Young's modulus of endocuticle along the major axis of the macrofibers is much stiffer than ${ }^{885}$ either of the other regions, at $8.47 \mathrm{GPa}$; however, Young's moduli for endocuticle along axes 2 and 3 are substantially lower, at $0.52 \mathrm{GPa}$. Due to this anisotropy, cuticular stiffness will depend upon the stacking angle of the balken layers (Reddy, 2004).

We note that the values for several constants were extracted from relevant literature, as we were unable to accurately measure these quantities in the species considered herein. These values do not necessarily represent the actual values present in $C$. longinasus, and we consider these 895 estimates as useful and necessary for the construction of this model, while considering the possible error introduced by their inclusion. We therefore investigate the potential error introduced by the following variables: (1) Young's modulus of fiber proteins, $E_{F P} ;(2)$ Poisson's ratio of fiber900

\footnotetext{
${ }^{6}$ The formulation of $Q$ presented in Eqs. 29 assumes that the longitudinal axis of the inhomogeneities is aligned with global axis 3. We therefore rotate the resultant tensor to align the macrofibers with global axis 1 , and assign the transverse normal to global axis $3 .^{905}$
}

Young's Moduli of $C$. longinasus Cuticle

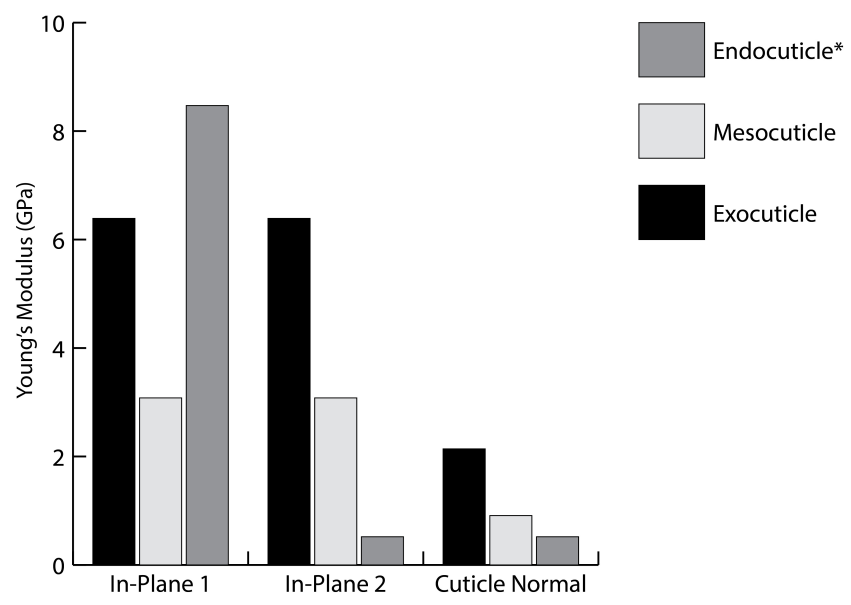

Figure 6: Summary of Young's moduli of elasticity along each global axis for each region of cuticle.

$\nu_{F P},(3)$ Young's modulus of matrix proteins, $E_{P M}$; (4) Poisson's ratio of matrix proteins, $\nu_{P M}$; (5) volume fraction of chitin-protein nanofibrils, $\varphi_{N F}$, and; $(6)$ volume fraction of pore canals, $\varphi_{P C}$. We do not examine the volume fraction of $\alpha$-chitin in a nanofibril, which was investigated by Nikolov et al. (2010), and found to have minimal effects on the resultant macroscopic elastic moduli with relatively large variation.

In Fig. 7, we graphically represent estimates of the macroscopic Young's moduli of each cuticular layer as these parameters change individually, with all others kept constant. The in-plane macroscopic Young's moduli exhibit little or no change with respect to the elastic constants of the fiber and matrix proteins, given the range of values used in the model. We observe that the structural arrangement of the chitin-protein nanofibrils has a strong influence on the resulting macroscopic properties of the cuticle, and influences which factors will contribute most to the overall stiffness of each region. For instance, the Bouligand structure greatly reduces the anisotropy of the cuticle compared to the parallel bundles of chitin in the endocuticle; increasing the volume fraction of nanofibrils in the mesocuticle to levels seen in the exocuticle results in an increase of the in-plane Young's modulus of about $3 \mathrm{GPa}$, while doing the same for the endocuticle results in an increase of almost $5 \mathrm{GPa}$ (along axis 1). Due to the parallel arrangement of chitin fibers in the endocuticle, smaller variations in the volume fraction of nanofibrils or pores elicit a larger change in the longitudinal Young's modulus compared to either mesocuticle or exocuticle.

The Bouligand structure is also responsible for the sensitivity of the normal Young's moduli to the properties of the fiber proteins. In Fig. 8 we present the point-wise elasticity of the model with respect to each of these variables. We observed that the macroscale Young's moduli are most sensitive to the volume fraction of nanofibrils (transverse) 
Table 1: Summary of elastic constants for the layers of cuticle. Young's and bulk moduli are given in GPa.

\begin{tabular}{ccccc}
\cline { 1 - 3 } Constant & & Exocuticle & Mesocuticle & Endocuticle* \\
\cline { 1 - 1 } \cline { 5 - 5 }$E_{1}$ & & 6.4 & 3.1 & 8.5 \\
$E_{2}$ & & 6.4 & 3.1 & 0.52 \\
$E_{3}$ & & 2.1 & 0.91 & 0.52 \\
\cline { 1 - 1 }$G_{12}$ & & 2.5 & 1.2 & 0.17 \\
$G_{31}$ & & 0.15 & 0.22 & 0.17 \\
$G_{23}$ & & 0.15 & 0.22 & 0.16 \\
\cline { 1 - 1 }$\nu_{12}$ & & 0.36 & 0.37 & 0.49 \\
$\nu_{21}$ & & 0.36 & 0.37 & 0.03 \\
$\nu_{23}$ & & 0.54 & 0.42 & 0.63 \\
$\nu_{32}$ & & 0.18 & 0.13 & 0.63 \\
$\nu_{31}$ & 0.18 & 0.13 & 0.06 \\
$\nu_{13}$ & 0.54 & 0.42 & 0.42 \\
\cline { 1 - 3 } & & & &
\end{tabular}

*Single layer of chitin macrofibers

Table 2: Homogenization schema used for cuticle layers of $C$. longinasus.

\begin{tabular}{|c|c|c|c|}
\hline RVE & Exocuticle & Mesocuticle & Endocuticle* \\
\hline Chitin Nanofibril & Mori-Tanaka & Mori-Tanaka & Mori-Tanaka \\
\hline Single Lamina & Effective Field & Mori-Tanaka & - \\
\hline Laminae sans Pores & Voigt Estimate & Voigt Estimate & — \\
\hline Laminae with Pores & Non-Interaction & Non-Interaction & - \\
\hline Chitin Macrofiber & - & - & Effective Field \\
\hline Porous Protein Matrix & - & - & Non-Interaction \\
\hline Balken Layer & - & - & Maxwell Scheme \\
\hline
\end{tabular}

$$
\begin{gathered}
C_{X}=\left[\begin{array}{cccccc}
9.5 & 4.7 & 2.6 & 0 & 0 & 0 \\
4.7 & 9.5 & 2.6 & 0 & 0 & 0 \\
2.6 & 2.6 & 3.1 & 0 & 0 & 0 \\
0 & 0 & 0 & 0.15 & 0 & 0 \\
0 & 0 & 0 & 0 & 0.15 & 0 \\
0 & 0 & 0 & 0 & 0 & 2.5
\end{array}\right] \\
C_{M}=\left[\begin{array}{cccccc}
4.1 & 1.80 & 0.74 & 0 & 0 & 0 \\
1.8 & 4.1 & 0.74 & 0 & 0 & 0 \\
0.74 & 0.74 & 1.1 & 0 & 0 & 0 \\
0 & 0 & 0 & 0.22 & 0 & 0 \\
0 & 0 & 0 & 0 & 0.22 & 0 \\
0 & 0 & 0 & 0 & 0 & 1.2
\end{array}\right] \\
C_{B}=\left[\begin{array}{cccccc}
9.0 & 0.63 & 0.60 & 0 & 0 & 0 \\
0.63 & 0.86 & 0.53 & 0 & 0 & 0 \\
0.60 & 0.53 & 0.86 & 0 & 0 & 0 \\
0 & 0 & 0 & 0.16 & 0 & 0 \\
0 & 0 & 0 & 0 & 0.17 & 0 \\
0 & 0 & 0 & 0 & 0 & 0.17
\end{array}\right]
\end{gathered}
$$



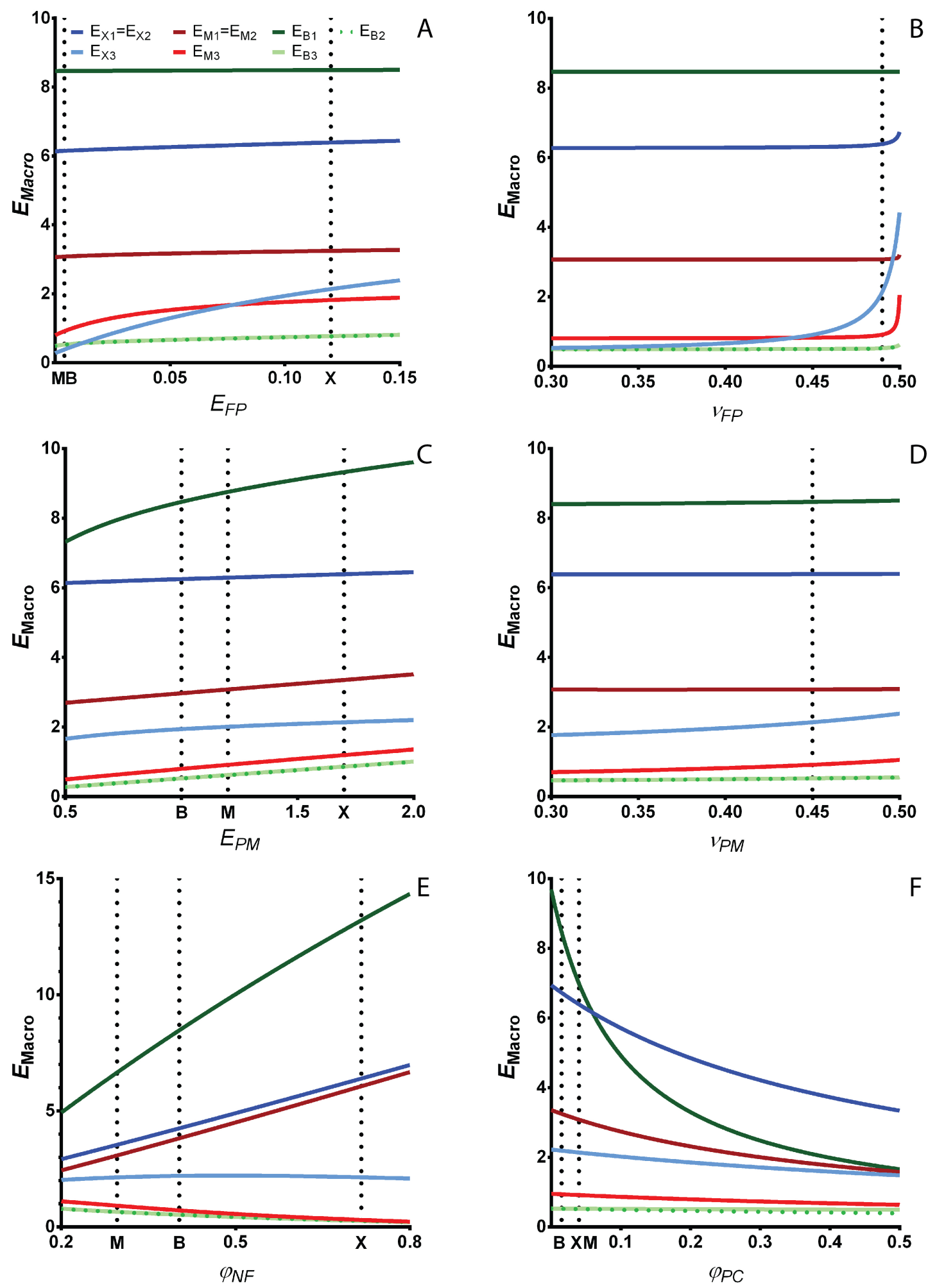

Figure 7: Macroscopic Young's moduli for exo-, meso-, and endocuticle (as indicated by subscripts X, M, B, respectively), along transverse axes 1 and 2, and normal axis 3, given variation in: A fiber protein modulus $\mathbf{B}$ fiber protein Poisson's ratio $\mathbf{C}$ protein matrix modulus $\mathbf{D}$ protein matrix Poisson's ratio $\mathbf{E}$ volume fraction of nanofibrils $\mathbf{F}$ volume fraction of pore canals.

*Vertical lines are placed at values used in the model, for each cuticle region, as indicated; a single, unlabeled line indicates that the same value was used for all regions 
$E_{X 1,2}$

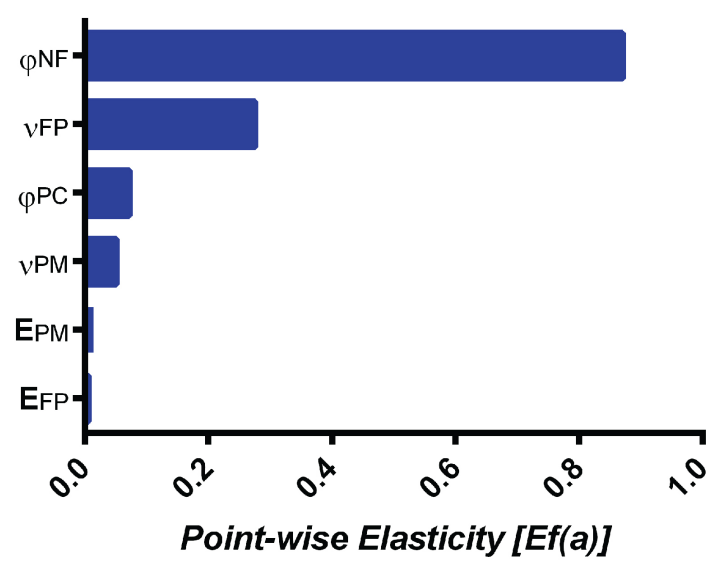

$E_{M 1,2}$

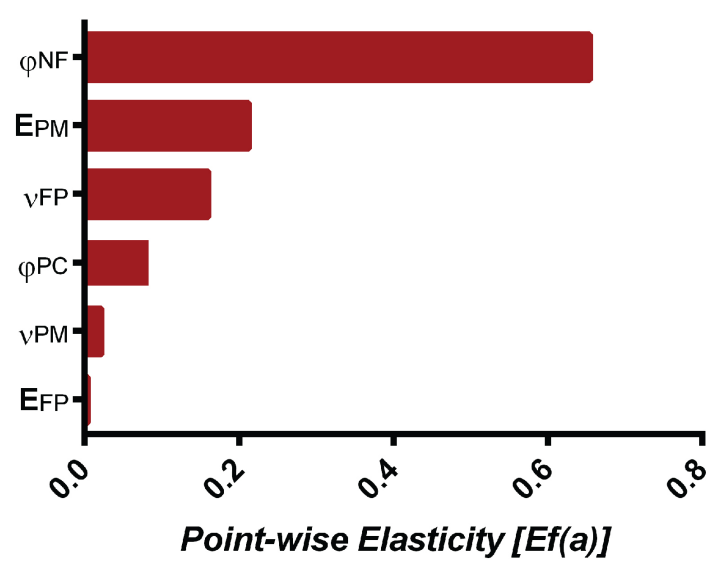

$E_{B 1}$

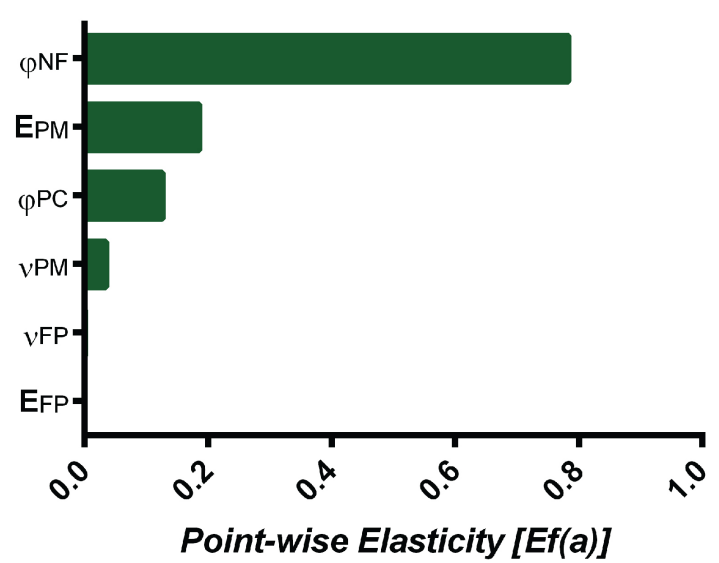

$E_{X 3}$

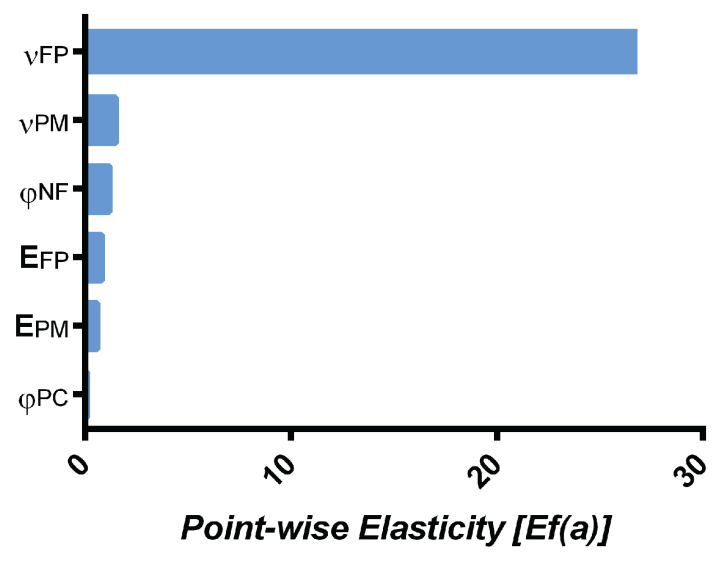

$E_{M 3}$

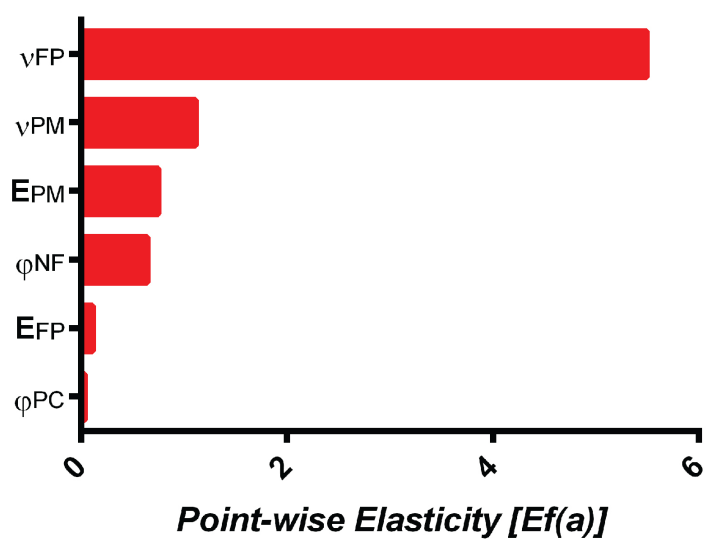

$E_{B 2,3}$

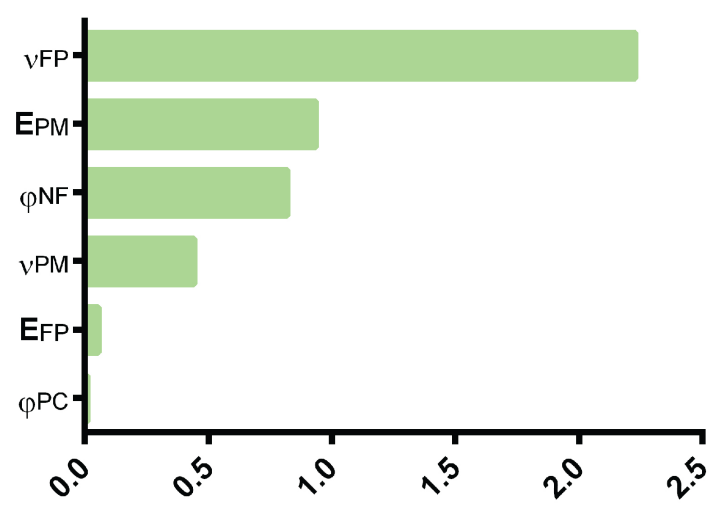

Point-wise Elasticity [Ef(a)]

Figure 8: Point-wise elasticity of the macroscopic Young's moduli for exo-, meso-, and endocuticle (as indicated by subscripts X, M, B, respectively), along transverse axes 1 and 2 , and normal axis 3, with respect to literature extracted parameters. Elasticity is highest with respect to Poisson's ratio of the fiber proteins (for normal moduli) and the volume fraction of nanofibrils (for transverse moduli). 
and Poisson's ratio of the fiber proteins (normal). Due to960 the significantly denser arrangement of the chitin-protein nanofibrils, the normal Young's modulus of the exocuticle is highly sensitive to changes in Poisson's ratio of the fiber proteins, with an elasticity of 26.8, as compared to mesocuticle (5.5) and endocuticle (2.2). However, given the com-965 paratively low elasticities of the normal Young's moduli for the other variables (the highest value is for exocuticle at 1.5 for $\nu_{P M}$ ), and the transverse Young's moduli to all infer that the model is generally quite robust over a wide970 range of input conditions. This observation corroborates the findings of Nikolov et al. (2011), who came to a similar conclusion.

\section{Discussion}

\subsection{Major Insights}

Our analysis indicates that the substantial differences between the regions of cuticle along the $C$. longinasus rostrum appear to be largely due to structural modifications, ather than chemical/compositional changes. In particular, the structural arrangement and volume fraction of the chitin nanofibrils appear to have the greatest influence on the macroscale properties of the corresponding cuticular region. We observed that significant variation in cer- ${ }^{985}$

${ }_{930}$ tain properties, such as the elastic modulus of the protein matrix, have comparatively little effect on the macroscale moduli. These results are largely consistent with previous studies that demonstrate the importance of microstructure in determining the macroscopic properties of bio- ${ }^{990}$ aterials (Cheng et al., 2009; Nikolov et al., 2011, 2010; Vincent and Wegst, 2004). Furthermore, as noted in Section 3.5, the endocuticle is much stiffer along axis 1 (corresponding to the longitudinal axis of the macrofibers) than either the exocuticle or mesocuticle. By contrast, in other ${ }^{95}$ arthropods, where the innermost region of the cuticle also conforms to the Bouligand structure, the endocuticle is often the most compliant region (Klocke and Schmitz, 2011; Nikolov et al., 2011, 2010). While the stiffness of beetle endocuticle is balanced by the comparatively low Young's modulus along the other axes, the stiffness of any cuticular structure in beetles is most likely dependent upon the stacking angle of the balken layers, due to the high degree of anisotropy of this material.

Additionally, we notice an inverse correlation between the in-plane and normal Young's moduli of the exocuti ${ }^{1005}$ cle and mesocuticle, with respect to fiber fraction. This is a result of the high degree of anisotropy of the chitinprotein nanofibrils, the effect of which is even more pronounced in the endocuticle. Within the chitin macrofibers, the properties of the protein matrix largely determine the macroscale moduli normal to the longitudinal axis (axis 1), especially at lower volume fractions of nanofibrils. As the volume fraction of nanofibrils increases, we observe a strong decrease in these moduli, as the chitin nanofibrils are significantly more compliant than the protein matrix along axes 2 and 3, while simultaneously an increase in stiffness is seen along axis 1 . In the exocuticle and mesocuticle, this effect is somewhat mitigated by the arrangement of nanofibrils in the Bouligand structure, which results in a degree of averaging of the moduli in the 1 and 2 axes. In general, the mesocuticle and endocuticle are of similar composition; however, the Bouligand structure creates a greater degree of isotropy in the mesocuticle due to the lower volume fraction of chitin.

How then do our outcomes explain the differences in elasticity between exocuticle and mesocuticle? We observe that differences between the elastic constants of exocuticle and mesocuticle are primarily correlated with the volume fraction of nanofibrils and the elastic properties of the fiber proteins. For the in-plane moduli, the volume fraction of nanofibrils is the primary contributing factor of the increased stiffness of the exocuticle, which is not strongly affected by variation of other parameters. In contrast, we note that the properties of the fiber proteins have a strong influence on the elastic constants of the exocuticle in the normal direction, due to the high volume fraction of the nanofibrils. The values of Young's modulus and Poisson's ratio of the fiber proteins must therefore be carefully considered, due to their influence on the properties of the exocuticle in the normal direction.

If we presume that these values are the same for both exo- and mesocuticle, then the resulting exocuticle would be significantly weaker in the normal direction than the mesocuticle. Based on the data reviewed in the work of Klocke and Schmitz (2011), this is unlikely for insects. We instead consider the reduced water content and increased cross-linking between nanofibrils and matrix (due to sclerotization), as well as the work of Vincent (1982); Vincent and Wegst (2004)and Ker (1977), in estimating Young's modulus and Poisson's ratio of the fiber proteins. This enables us to utilize more appropriate and realistic values that account for these factors, and provide results that closely match what is observed in insects ${ }^{7}$ [i.e., the exocuticle is stiffer than the mesocuticle in the normal direction (Klocke and Schmitz, 2011)]. Further investigation is needed to more accurately determine these parameters for members of the genus Curculio.

\subsection{Modeling Limitations}

Our model suffers from two critical limitations. The first is the pragmatic constraint of approximating certain values based on the literature. Generally, these values can vary by nearly an order of magnitude without creating a correspondingly large variation in the model output. We are also able to mitigate the potential for significant error

\footnotetext{
${ }^{7}$ It is interesting to consider that increasing the fiber fraction of the exocuticle results in transverse (in-plane) stiffening at the expense of stiffness in the normal direction. We speculate that sclerotization might occur more strongly in the exocuticle to counter this effect, and thereby increase stiffness in the normal direction.
} 
${ }_{1010}$ by comparing the results of the model to the known elastic properties of insect cuticle. The second limitation concerns the scope of the model, which is restricted to conditions of small strains and linear-elastic mechanical behavior. The viscoelastic and viscoplastic behavior of the cuticle dur-

1015 ing significant deformation has been explored in lobster cuticle, but has not been accurately described by any micromechanical model, mainly because of the numerous and difficult-to-measure factors that require assessment in order to accurately formulate such a model. For example, we would require a full characterization of the makeup of the protein matrix, degree of interconnection between chitin fibers, and presence of interply fibers (Nikolov et al., 2011; Sachs et al., 2008; van de Kamp et al., 2015). In spite of these limitations, our model yields useful insights into the interplay of structural and compositional factors in relation to the macroscale properties of each region of cuticle. Specifically, we provide a predictive framework within which we may systematically compare the optimization of the female $C$. longinasus cuticle in response to the mechanical stresses to that of different species in this genus, as well as in other phylogenetically related groups. Refining our approach to accommodate experimentally vali $\overline{1}_{\overline{1} 090}$ dated data for multiple species can produce novel insights into the evolutionary and developmental trade-offs of this system.

Future research should focus in particular on the $\mathrm{de}_{\overline{1} 095}$ gree of optimization that has occurred in related Curculio species in response to mechanical stress, and consider the evolutionary trajectory of this genus as a whole. The elastic constants generated by our approach can now be ap ${ }_{1100}$ plied to full-scale three-dimensional models of the head of C. longinasus. In conjunction with data about the orientation angles of the endocuticular macrofibers in this species, these constants will permit us to perform finite $\mathrm{el}_{\mathbf{1 1 0 5}}$ 45 ement analysis to simulate mechanical loads encountered by females during oviposition. By examining the responses of different species and the localization of stress and strain on their heads during deformation, and aligning these vari ${ }_{+110}$ ations phylogenetically, we hope to better mechanistically understand the process of structural adaptation and optimization that has occurred during the evolution of this group of weevils (Toju, 2008; Toju and Sota, 2006a,b). ${ }_{1115}$

\section{Acknowledgments}

This research was funded in part by the National Sci ${ }^{1120}$ ence Foundation (DEB-1155984) and the United States Department of Agriculture Agricultural Research Service (Agreement 58-1275-1-335).

\section{References}

Aguirre Uribe, L.A., 1978. Biology of the immature stages of the pecan weevil Curculio caryae (Horn) and oviposition habits of the $\mathrm{e}^{130}$ adult weevil. Ph.D. thesis. Texas A \& M University.
Benveniste, Y., 1987. A new approach to the application of MoriTanaka's theory in composite materials. Mech. Mater. 6, 147-157. doi:10.1016/0167-6636(87)90005-6.

Blackwell, J., Weih, M., 1980. Structure of chitin-protein complexes: ovipositor of the ichneumon fly Megarhyssa. J. Mol. Biol. 137, 49-60. doi:10.1016/0022-2836 (80)90156-4.

Bouligand, Y., 1972. Twisted fibrous arrangements in biological materials and cholesteric mesophases. Tissue Cell 4, 189-217. doi:10.1016/S0040-8166 (72) 80042-9.

Buryachenko, V.a., Kushch, V.I., Dudka, V.a., Roy, A., 2008. Effective stiffness of composites reinforced by cylindrical fibers with smooth ends, with potential application to nanocomposites. Acta Mech. 198, 129-146. doi:DOI10.1007/s00707-007-0531-z.

Chen, P.Y., Lin, A.Y.M., McKittrick, J., Meyers, M.A., 2008. Structure and mechanical properties of crab exoskeletons. Acta Biomater. 4, 587-596. doi:10.1016/j.actbio.2007.12.010.

Cheng, L., Wang, L., Karlsson, A.M., 2008. Image analyses of two crustacean exoskeletons and implications of the exoskeletal microstructure on the mechanical behavior. J. Mater. Res. 23, 2854 2872. doi:10.1557/JMR . 2008.0375.

Cheng, L., Wang, L., Karlsson, A.M., 2009. Mechanics-based analysis of selected features of the exoskeletal microstructure of Popillia japonica. J. Mater. Res. 24, 3253-3267. doi:10.1557/jmr. 2009. 0409.

Davis, S.R., 2014. Morphology, phylogeny, and evolutionary development in the weevils (Insecta: Coleoptera: Curculionoidea). Ph.D. thesis. University of Kansas.

Doghri, I., 2000. Mechanics of deformable solids: linear, nonlinear, analytical, and computational aspects. Springer, New York; Berlin.

Dumont, E.R., Grosse, I.R., Slater, G.J., 2009. Requirements for comparing the performance of finite element models of biological structures. J. Theor. Biol. 256, 96-103. doi:10.1016/j.jtbi. 2008. 08.017.

Flannigan, W.C., 1998. Finite Element Modeling of Arthropod Exoskeleton. Ph.D. thesis. Case Western University.

Gibson, L.P., 1969. Monographof the genus Curculio in theNew World (Coleoptera: Curculionidae). Part I. United States and Canada. Misc. Publ. Entomol. Soc. Am. 6, 239-285.

Giraud-Guille, M.M., Chanzy, H., Vuong, R., 1990. Chitin crystals in arthropod cuticles revealed by diffraction contrast transmission electron microscopy. J. Struct. Biol. 103, 232-240. doi:10.1016/ 1047-8477 (90) 90041-A.

Hepburn, H.R., Ball, A., 1973. On the structure and mechanical properties of beetle shells. J. Mater. Sci. 8, 618-623.

Herbert, R.C., Young, P.G., Smith, C.W., Wootton, R.J., Evans, K.E., 2000. The hind wing of the desert locust (Schistocerca gregaria Forskal). III. A finite element analysis of a deployable structure. J. Exp. Biol. 203, 2945-2955.

Hill, R., 1952. The Elastic Behaviour of a Crystalline Aggregate. Proc. Phys. Soc. Sect. A 65, 349-354. doi:10.1088/0370-1298/ $65 / 5 / 307$.

Hörnschemeyer, T., Bond, J., Young, P.G., 2013. Analysis of the functional morphology of mouthparts of the beetle Priacma serrata, and a discussion of possible food sources. J. Insect Sci. 13, 126. doi:10.1673/031.013.12601.

Hughes, J., Vogler, A.P., 2004. The phylogeny of acorn weevils (genus Curculio) from mitochondrial and nuclear DNA sequences: The problem of incomplete data. Mol. Phylogenet. Evol. 32, 601-615. doi:10.1016/j . ympev. 2004.02.007.

Hyun, S., Torquato, S., 2000. Effective elastic and transport properties of regular honeycombs for all densities. J. Mater. Res. 15, 1985-1993. doi:10.1557/JMR . 2000.0285.

Kachanov, M., 1999. Solids with cracks and non-spherical pores : proper parameters of defect density and effective elastic properties. Int. J. Fract. 97, 1-32.

Kachanov, M., Sevostianov, I., 2005. On quantitative characterization of microstructures and effective properties. Int. J. Solids Struct. 42, 309-336. doi:10.1016/j.ijsolstr.2004.06.016.

van de Kamp, T., Greven, H., 2010. On the architecture of beetle elytra. Entomol. Heute 22, 191-204. 
van de Kamp, T., Riedel, A., Greven, H., 2015. Arthropod Structure \& Development Micromorphology of the elytral cuticle of bee 205 tles, with an emphasis on weevils (Coleoptera : Curculionoidea ). Arthropod Struct. Dev. 45, 14-22. URL: http://dx.doi.org/ 10.1016/j.asd.2015.10.002, doi:10.1016/j.asd.2015.10.002.

Kaya, M., Lelesius, E., Nagrockaite, R., Sargin, I., Arslan, G., Mol, A., Baran, T., Can, E., Bitim, B., 2015. Differentiations of Chitin210 Content and Surface Morphologies of Chitins Extracted from Male and Female Grasshopper Species. PLoS One 10, e0115531. doi:10. 1371/journal. pone.0115531.

Ker, R.F., 1977. Some structural and mechanical properties of locust and beetle cuticle. Ph.D. thesis. University of Oxford.

Klocke, D., Schmitz, H., 2011. Water as a major modulator of the mechanical properties of insect cuticle. Acta Biomater. 7, 29352942. doi:10.1016/j.actbio.2011.04.004.

Leopold, R.A., Newman, S.M., Helgeson, G., 1992. A comparison of cuticle deposition during the pre- and posteclosion stages of the220 adult weevil, Anthonomus grandis Boheman (Coleoptera : Curculionidae). doi:10.1016/0020-7322(92)90004-7.

Mccullough, E.L., Tobalske, B.W., Emlen, D.J., 2014. Structural adaptations to diverse fighting styles in sexually selected weapons. Proc. Natl. Acad. Sci. 2014. doi:10.1073/pnas.1409585111.

McNamara, M.E., Briggs, D.E.G., Orr, P.J., Noh, H., Cao, H., 2012. The original colours of fossil beetles. Proc. R. Soc. B Biol. Sci. 279, 1114-1121. doi:10.1098/rspb.2011.1677.

Moffett, M., 1989. Life in a Nutshell. Natl. Geogr. Mag. 176, 783784.

1160 Mori, T., Tanaka, K., 1973. Average stress in matrix and average elastic energy of materials with misfitting inclusions. Acta Metall. 21, 571-574. doi:10.1016/0001-6160(73)90064-3.

Morimoto, K., Kojima, H., 2003. Morphologic Characters of the Weevil Head and Phylogenetic Implications (Coleoptera, Cur $¥ 235$ culionoidea). Esakia 43, 133 - 169

Mura, T., 1987. Micromechanics of defects in solids. volume 3. M. Nijhoff, Dordrecht; Higham, MA; Boston.

Neville, A.C., Parry, D.A., Woodhead-Galloway, J., 1976. The chitin crystallite in arthropod cuticle. J. Cell Sci. 21, 73-82.

Nikolov, S., Fabritius, H., Petrov, M., Friák, M., Lymperakis, L. Sachs, C., Raabe, D., Neugebauer, J., 2011. Robustness and optimal use of design principles of arthropod exoskeletons studied by ab initio-based multiscale simulations. J. Mech. Behav. Biomed. Mater. 4, 129-145. doi:10.1016/j.jmbbm.2010.09.015.

Nikolov, S., Petrov, M., Lymperakis, L., Friák, M., Sachs, C., Fabritius, H.O., Raabe, D., Neugebauer, J., 2010. Revealing the design principles of high-performance biological composites using $\mathrm{Ab}$ initio and multiscale simulations: The example of lobster cuticle. Adv. Mater. 22, 519-526. doi:10.1002/adma. 200902019.

Oberprieler, R., Anderson, R., Marvaldi, A., 2014. Curculionoidea Latreille, 1802: Introduction, Phylogeny., in: Leschen, R., Beutel, R. (Eds.), Handb. Zool. Arthropoda Insecta Coleopt. Vol. 3 Morphol. Syst.. De Gruyter, Berlin. chapter 3, pp. 503-522.

Raabe, D., Sachs, C., Romano, P., 2005. The crustacean exoskeleton as an example of a structurally and mechanically graded biologica nanocomposite material. Acta Mater. 53, 4281-4292. doi:10. 1016/j.actamat. 2005.05.027.

Reddy, J.N., 2004. Mechanics of laminated composite plates and shells: theory and analysis. volume 2nd. CRC Press, London; Boca Raton, FL.

Saadat, F., Sevostianov, I., Giraud, A., 2012. Approximate representation of a compliance contribution tensor for a cylindrical inhomogeneity normal to the axis of symmetry of a transversely isotropic material. Int. J. Fract. 174, 237-244. doi:10.1007/ s10704-012-9688-0.

Sachs, C., Fabritius, H., Raabe, D., 2008. Influence of microstructure on deformation anisotropy of mineralized cuticle from the lobster Homarus americanus. J. Struct. Biol. 161, 120-132. doi:10.1016/ j.jsb.2007.09.022.

Sevostianov, I., 2014. On the shape of effective inclusion in the Maxwell homogenization scheme for anisotropic elastic composites. Mech. Mater. 75, 45-59. doi:10.1016/j.mechmat.2014.03. 003.
Sevostianov, I., Giraud, A., 2013. Generalization of Maxwell homogenization scheme for elastic material containing inhomogeneities of diverse shape. Int. J. Eng. Sci. 64, 23-36. doi:10.1016/j. ijengsci.2012.12.004

Sevostianov, I., Kachanov, M., 2002. Explicit cross-property correlations for anisotropic two-phase composite materials. J. Mech. Phys. Solids 50, 253-282. doi:10.1016/S0022-5096(01) 00051-5.

Sevostianov, I., Kachanov, M., 2008. On approximate symmetries of the elastic properties and elliptic orthotropy. Int. J. Eng. Sci. 46, 211-223. doi:10.1016/j.ijengsci.2007.11.003.

Sevostianov, I., Levin, V., Kachanov, M., 2001. On the modeling and design of piezocomposites with prescribed properties. Arch. Appl. Mech. (Ingenieur Arch. 71, 733-747. doi:10.1007/s004190100181.

Sevostianov, I., Levin, V., Radi, E., 2015. Effective properties of linear viscoelastic microcracked materials: Application of Maxwell homogenization scheme. Mech. Mater. 84, 28-43. doi:10.1016/j . mechmat.2015.01.004.

Sevostianov, I., Yilmaz, N., Kushch, V., Levin, V., 2005. Effective elastic properties of matrix composites with transverselyisotropic phases. Int. J. Solids Struct. 42, 455-476. doi:10.1016/ j.ijsolstr.2004.06.047.

Ting, P.C., 1933. Feeding mechanisms of weevils, their function, and relationship to classification. Mon. Bull. Dep. Agric. State Calif. $22,161-165$.

Ting, P.C., 1936. The mouth parts of the coleopterous group Rhynchophora. Microentomology 1, 93-114.

Toju, H., 2008. Fine-scale local adaptation of weevil mouthpart length and camellia pericarp thickness: altitudinal gradient of a putative arms race. Evolution 62, 1086-1102. doi:10.1111/j. 1558-5646.2008.00341.x.

Toju, H., Sota, T., 2006a. Adaptive divergence of scaling relationships mediates the arms race between a weevil and its host plant. Biol. Lett. 2, 539-542. doi:10.1098/rsbl.2006.0514.

Toju, H., Sota, T., 2006b. Imbalance of predator and prey armament: geographic clines in phenotypic interface and natural selection. Am. Nat. 167, 105-117. doi:10.1086/498277.

Vilchevskaya, E., Sevostianov, I., 2015. Effective elastic properties of a particulate composite with transversely-isotropic matrix. Int. J. Eng. Sci. 94, 139-149. doi:10.1016/j.ijengsci.2015.05.006.

Vincent, J.F.V., 1982. Structural biomaterials. Halsted Press, New York.

Vincent, J.F.V., Wegst, U.G.K., 2004. Design and mechanical properties of insect cuticle. Arthropod Struct. Dev. 33, 187-199. doi:10.1016/j.asd.2004.05.006. 